\title{
Hydrogen and Coordination Bonding Supramolecular Structures of Trimesic Acid on $\mathrm{Cu}(110)^{\dagger}$
}

\author{
Thomas Classen, Magalí Lingenfelder, Yeliang Wang, Rishav Chopra, Chariya Virojanadara, \\ Ulrich Starke, and Giovanni Costantini*,:
}

Max-Planck-Institut für Festkörperforschung, Heisenbergstrasse 1, D-70569 Stuttgart, Germany

\author{
Guido Fratesi, Stefano Fabris, Stefano de Gironcoli, and Stefano Baroni \\ INFM-CNR DEMOCRITOSTheory@Elettra group and SISSA Scuola Internazionale Superiore di Studi \\ Avanzati, via Beirut 2-4, I-34014 Trieste, Italy
}

\author{
Sam Haq and Rasmita Raval \\ Surface Science Research Centre, University of Liverpool, Liverpool L69 3BX, U.K.
}

\begin{abstract}
Klaus Kern
Max-Planck-Institut für Festkörperforschung, Heisenbergstrasse 1, D-70569 Stuttgart, Germany, and Institut de Physique des Nanostructures, Ecole Polytechnique Fédérale de Lausanne, CH-1015 Lausanne, Switzerland
\end{abstract}

Received: July 30, 2007; In Final Form: October 8, 2007

\begin{abstract}
The adsorption of trimesic acid (TMA) on $\mathrm{Cu}(110)$ has been studied in the temperature range between 130 and $550 \mathrm{~K}$ and for coverages up to one monolayer. We combine scanning tunneling microscopy (STM), low-energy electron diffraction (LEED), reflection absorption infrared spectroscopy (RAIRS), X-ray photoemission spectroscopy (XPS), and density functional theory (DFT) calculations to produce a detailed adsorption phase diagram for the $\mathrm{TMA} / \mathrm{Cu}(110)$ system as a function of the molecular coverage and the substrate temperature. We identify a quite complex set of adsorption phases, which are determined by the interplay between the extent of deprotonation, the intermolecular bonding, and the overall energy minimization. For temperatures up to $280 \mathrm{~K}$, TMA molecules are only partly deprotonated and form hydrogen-bonded structures, which locally exhibit organizational chirality. Above this threshold, the molecules deprotonate completely and form supramolecular metal-organic structures with $\mathrm{Cu}$ substrate adatoms. These structures exist in the form of single and double coordination chains, with the molecular coverage driving distinct phase transitions.
\end{abstract}

\section{Introduction}

The self-organization of molecules at surfaces into larger assemblies offers a promising way to fabricate functional nanostructures. ${ }^{1-3}$ Recently, there has been particular interest in creating supramolecular structures using small carboxylic acids, ${ }^{4}$ as the specific surface interactions of these molecules lead to the formation of highly ordered and regular organometallic networks with novel catalytic and magnetic properties. ${ }^{5}$

The chemisorption of small benzene-carboxylic acids on metal surfaces has been extensively studied using a range of surface-sensitive techniques, including, reflection absorption infrared spectroscopy (RAIRS) ${ }^{6-10}$ and X-ray photoelectron spectroscopy (XPS). ${ }^{1-14}$ It is well-established that on specific substrates, the acid groups can deprotonate to produce carboxylate $\left(\mathrm{COO}^{-}\right)$groups, ${ }^{7-12}$ which generally interact strongly with metal surfaces. They can also trap and bind with adatoms either intentionally co-deposited with the molecules or already existing

\footnotetext{
† Part of the "Giacinto Scoles Festschrift".

* To whom correspondence should be addressed. Telephone: 0049-7116891541. Fax: 0049-711-6891662.E-mail: gio@fkf.mpg.de.

$\doteqdot$ Present address: Department of Chemistry, University of Warwick, Gibbet Hill Road, Coventry CV47AL, United Kingdom.
}

as a 2D background "gas" that results from the emission of atoms from low coordination sites such as steps and kinks. ${ }^{7}$

In a recent series of experiments, it has been shown that small di- and tricarboxylic acids can be used to mimic 3D metalorganic coordination networks (MOCNs) $)^{15-23}$ in two dimensions at surfaces. ${ }^{5,24-43}$ The MOCN concept originates in coordination chemistry, where organic molecules are used as linkers and metal atoms as nodes to construct extended 3D hollow networks. Through the careful choice of starting materials, these networks can be tailored for specific magnetic or catalytic properties, as well as for other applications such as gas storage. ${ }^{19,44}$ The creation of MOCNs directly at surfaces is considered to be relevant for similar technological applications including information storage and processing ${ }^{5,40,45}$ and also constitutes a model system to study low-dimensional magnetism at surfaces. ${ }^{34,35}$

A typical linker molecule used for surface MOCNs is trimesic acid (TMA) comprised of three carboxylic acid groups ordered in a planar triangular arrangement around the central phenyl ring, Figure 1. TMA has been investigated in its acidic form in solids ${ }^{46,47}$ as a three-dimensional MOCN coordinated to $\mathrm{Cu}$ ions $^{23,48}$ and on low reactivity surfaces, ${ }^{12,14,49-55}$ where mostly hexagonal honeycomb networks are found, which are formed 


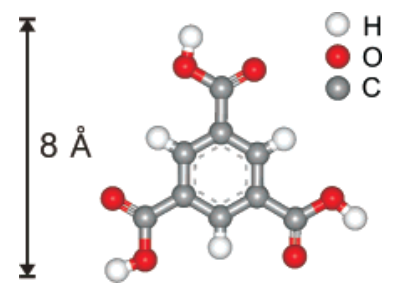

Figure 1. Model of the TMA molecule.

by intermolecular hydrogen bonding. However, in its deprotonated form, the carboxylate groups may combine with metal atoms to form extended metal-organic coordination networks in three dimensions in the solid state ${ }^{48,56,47}$ and on surfaces in two ${ }^{24-26,28}$ and one dimensions. ${ }^{34}$

We have used a combination of complementary experimental techniques to achieve complete insight into these surface networks. RAIRS is particularly useful for establishing the chemical identity, the local coordination modes, and the orientation of the surface acid/carboxylate groups. XPS can provide information about the chemical bond configuration within the molecule and the coordination to the substrate. However, the evaluation of the structural characteristics of the networks as well as their degree of order is outside the scope of vibrational or photoemission spectroscopy. Low-energy electron diffraction (LEED) and scanning tunneling microscopy (STM) are better suited for these measurements. These techniques were coupled to density functional theory (DFT) calculations to investigate the self-organization of TMA on $\mathrm{Cu}(110)$. The combination of the different results has allowed us to attain a detailed understanding of the adsorbate-substrate interactions and to reconstruct the complex adsorption phase diagram of the TMA/Cu(110) system as a function of the surface temperature and molecular coverage.

This paper has been organized to follow the evolution of molecular structures as a function of temperature and coverage. Below $280 \mathrm{~K}$, section III, organization largely results from $\mathrm{H}$ bonding between acid groups. With increasing temperature, this changes as carboxylate groups are formed, and above $280 \mathrm{~K}$, these latter groups combine with metal atoms to form metalorganic structures. These are described in sections IV and V for low and high coverage, respectively. Finally, in section VI, all of the different experimental and theoretical results are summarized and used for the construction of the TMA/Cu(110) adsorption phase diagram.

\section{Methods}

For the STM measurements, the $\mathrm{Cu}(110)$ single crystal was prepared in a standard ultrahigh vacuum (UHV) preparation chamber with a base pressure of less than $2 \times 10^{-10} \mathrm{mbar}$. The sample was cleaned using cycles of $\mathrm{Ar}^{+}$sputtering $(900 \mathrm{eV})$ and annealing to $850 \mathrm{~K}$. The TMA (Fluka Chemie AG, purity $>97 \%$ ) was degassed in a ceramic crucible for several days under UHV conditions before evaporation at $460 \mathrm{~K}$. The sample was transferred under UHV to a second chamber (base pressure of $6 \times 10^{-11}$ mbar) containing a variable-temperature STM. Unless otherwise stated, the STM measurements were performed at room temperature in constant current mode. Typical scanning conditions were a sample bias of $-1 \mathrm{~V}$ (filled state imaging) and a tunneling current of $1 \mathrm{nA}$.

The XPS experiments were carried out in a different UHV analysis chamber with a base pressure of $1 \times 10^{-10} \mathrm{mbar}$. The cleaning of the $\mathrm{Cu}(110)$ substrate and the evaporation of TMA were similar to those for the STM measurements. LEED was used to control and compare the structural evolution of the surface phases to the other experimental setups. A standard $\mathrm{X}$-ray source was used for $\mathrm{Mg} \mathrm{K} \alpha$ radiation excitation with a $45^{\circ}$ incidence angle. Core level emission spectra for carbon, oxygen, and copper $\left(\mathrm{C} 1 \mathrm{~s}, \mathrm{O} 1 \mathrm{~s}\right.$, and $\left.\mathrm{Cu} 2 \mathrm{p}_{3 / 2}\right)$ were acquired using a Phoibos 100 hemispherical analyzer (Specs $\mathrm{GmbH}$ ) at normal emission direction. The total energy resolution was set to $0.5-$ $1.0 \mathrm{eV}$. For the line shape analysis of the core level spectra, a fitting procedure was applied. ${ }^{58}$ The binding energies are referenced to the $\mathrm{Cu} 2 \mathrm{p}_{3 / 2}$ bulk component with a binding energy of $932.2 \mathrm{eV}$. Only for the case of low coverage and low temperature (section III) was a high-resolution XPS system (Kratos, Manchester) employed, which was equipped with a more intense X-ray source.

A third UHV setup was used for the RAIRS measurements. The sample preparation was similar to that described above. The TMA was evaporated from a small glass tube located in a differentially pumped housing, separate from the main chamber. The purity of the sublimed TMA was confirmed using mass spectrometry in addition to the IR fingerprint of a thick condensed film at $85 \mathrm{~K}$. The IR data was collected using a FTIR instrument interfaced to the main chamber using $\mathrm{KBr}$ windows and optics. A mercury-cadmium-telluride detector was used, which accesses a spectral range of $670-4000 \mathrm{~cm}^{-1}$. The spectra were ratioed against a reference spectrum of the clean surface measured prior to each experiment. They were collected at a resolution of $4 \mathrm{~cm}^{-1}$ with the coaddition of 256 scans. Also for this system, LEED measurements were used to calibrate TMA coverage and deposition rates.

The DFT calculations were carried out in the generalized gradient approximation (GGA) of Perdew-Burke-Ernzerhof ${ }^{59}$ in the pseudopotential plane wave framework (plane wave cutoff at $24 \mathrm{Ry}$ ) with ultrasoft pseudopotentials ${ }^{60}$ as implemented in the Quantum-espresso/PWscf ${ }^{61}$ computer package. A simplified three-layer slab model was employed for the $\mathrm{Cu}(110)$ surface. The surface Brillouin zone was sampled with meshes of special points corresponding to at least an $8 \times 8 \times 8$ grid for the bulk Brillouin zone. While the upper layer was relaxed, the distance between the other layers was kept fixed at the bulk value. $\mathrm{Cu}$ adatoms and deprotonated TMA molecules were positioned on the upper surface layer. Structural relaxation was carried out according to the Hellmann-Feynman forces. Simulated STM images were calculated by the Tersoff-Hamann method ${ }^{62}$ as a spatially resolved density of states (DOS) integrated in energy from a bias potential $(-1.0 \mathrm{eV})$ to the Fermi energy.

\section{TMA Deposition at Low Substrate Temperatures}

A. STM Measurements at $130 \mathrm{~K}$. When TMA is deposited onto the $\mathrm{Cu}(110)$ surface at $130 \mathrm{~K}$, STM shows triangular protrusions which are assigned to flat-lying TMA molecules. ${ }^{24-26,28,49,51,53}$ As confirmed by LEED, no long-range structural order is detected (see Figure 2), which is attributed to restricted surface diffusion at low substrate temperatures. Nevertheless, a short-range interaction between neighboring molecules is observed. A "tip-to-tip" alignment is often found in which the acid group at the "tip" of a TMA molecule points to the tip of a neighbor. This geometry is typical for dimeric hydrogen bonding between the acid groups. ${ }^{24}$ In addition, other possible H-bonding modes with "side-to-side" and "tip-to-side" attachments are also observed; see Figure 2. These STM observations suggest that at $130 \mathrm{~K}$, the acid groups of TMA are at least partially intact and that hydrogen bonding is the dominant intermolecular interaction at this temperature. ${ }^{24,49,51,53}$

B. IR Measurements. Figure 3 shows a series of infrared (IR) spectra as a function of increasing coverage during TMA 

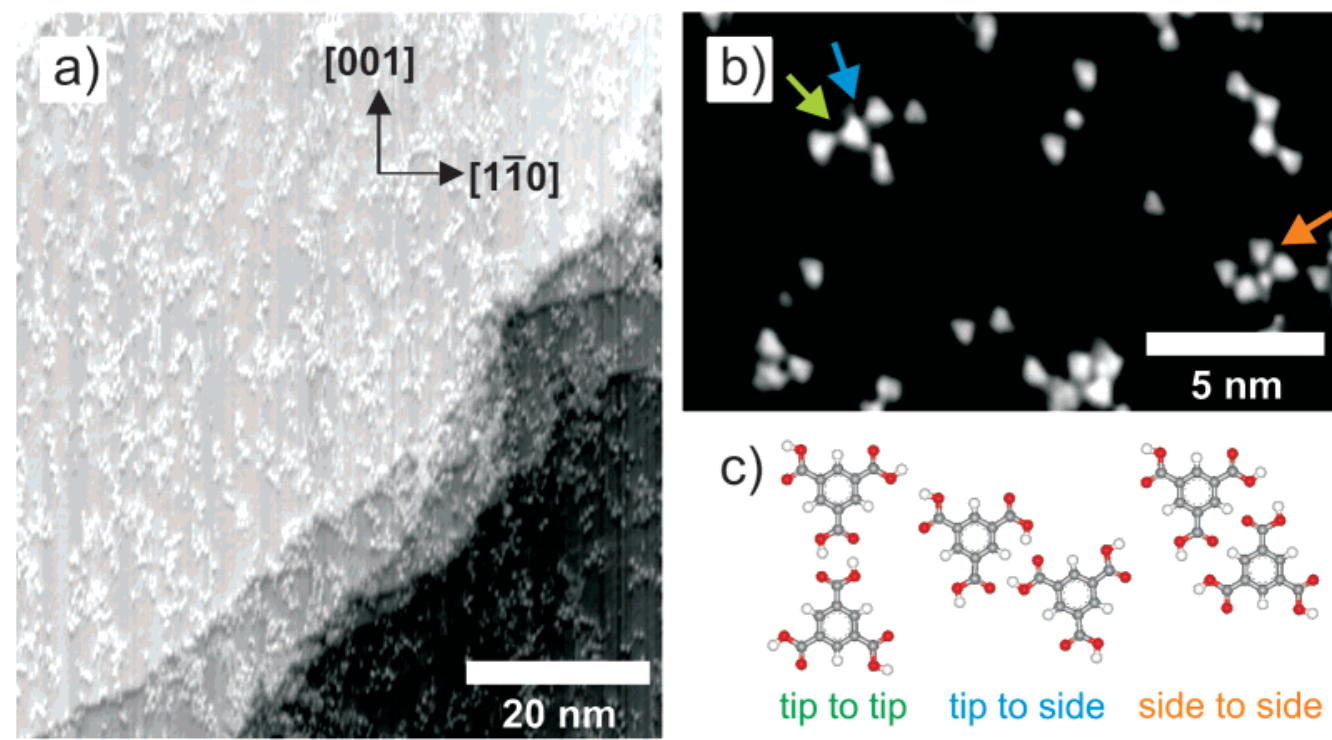

c)

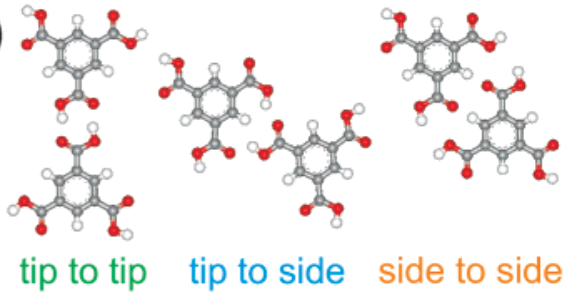

Figure 2. Adsorption of TMA on $\mathrm{Cu}(110)$ at $130 \mathrm{~K}$ (cooled to $40 \mathrm{~K}$ for measurement); (a) overview STM image, (b) magnified image with indication of commonly observed bonding motifs, and (c) corresponding schematic representations of such motifs. We note that these binding geometries would also be possible between partially deprotonated TMA molecules.

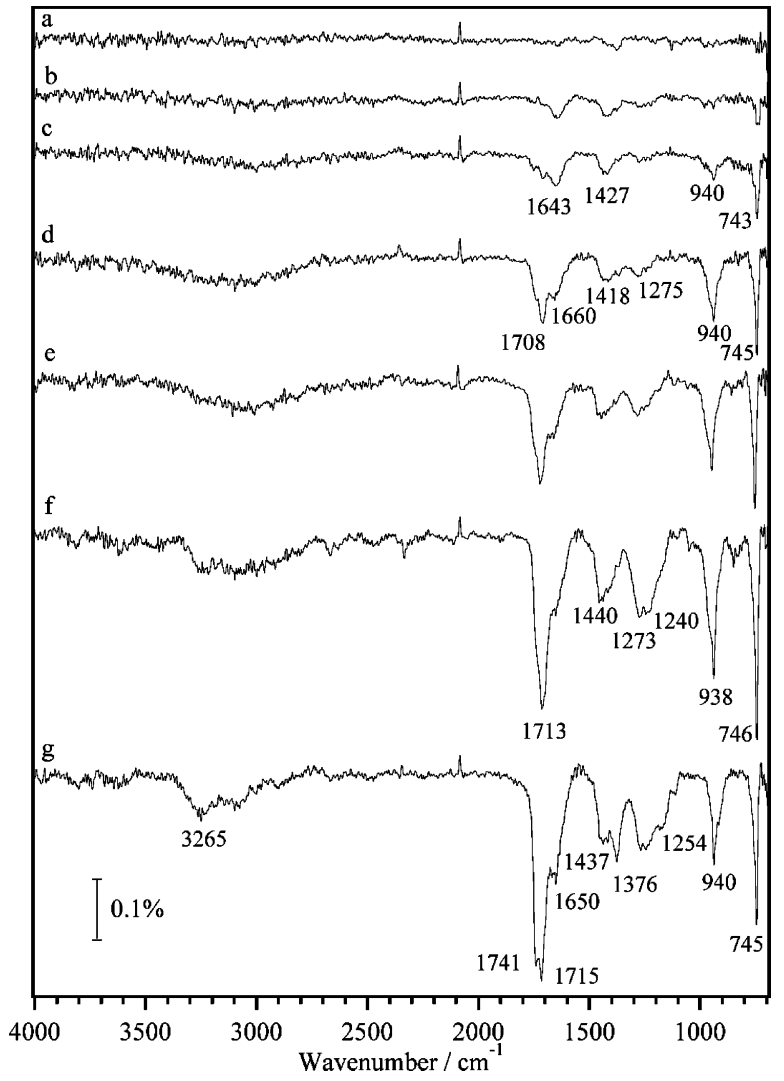

Figure 3. RAIRS measurements of $\mathrm{TMA} / \mathrm{Cu}(110)$ with increasing coverage at $85 \mathrm{~K}$. The vertical scale bar indicates the variation of signal strength with respect to the clean $\mathrm{Cu}(110)$ surface.

deposition on $\mathrm{Cu}(110)$ at $85 \mathrm{~K}$. For low coverage (spectra a-c), the main bands are found at 743, 940, 1427, and $1643 \mathrm{~cm}^{-1}$. Presumably, these are associated with species in the first monolayer. Subsequently, as the coverage increases (spectra $\mathrm{d}-\mathrm{g}$ ), there is a distinct change as additional bands appear at around 1240, 1440, 1713, and $3265 \mathrm{~cm}^{-1}$.

The absence of a published normal mode vibrational analysis for TMA adsorbed under UHV conditions requires the use of a number of different resources for the assignment of the infrared spectra shown in this work. These include the identification of
TABLE 1: Assignment of the Main Absorption Bands Experimentally Observed $^{a}$

\begin{tabular}{|c|c|c|c|}
\hline mode & description & direction & $\begin{array}{c}\text { frequency } \\
\mathrm{cm}^{-1}\end{array}$ \\
\hline \multicolumn{4}{|c|}{ Carboxylic Group } \\
\hline$v(\mathrm{OH})$ & $\mathrm{O}-\mathrm{H}$ stretch (free) & in-plane & 3572 \\
\hline$v(\mathrm{OH})$ & $\mathrm{O}-\mathrm{H}$ stretch (H-bonded) & in-plane & 3200 (broad) \\
\hline$v(\mathrm{C}=\mathrm{O})$ & $\mathrm{C}=\mathrm{O}$ stretch & in-plane & $1708-1765$ \\
\hline$v(\mathrm{C}-\mathrm{O})$ & $\mathrm{C}-\mathrm{O}$ stretch & in-plane & $1200-1300$ \\
\hline$\delta(\mathrm{COH})$ & $\mathrm{C}-\mathrm{O}-\mathrm{H}$ bend & in-plane & $1300-1400$ \\
\hline$v(\mathrm{C}-\mathrm{COOH})$ & $\mathrm{C}-\mathrm{C}$ stretch & in-plane & $1150-1200$ \\
\hline$\pi(\mathrm{OH})$ & $\mathrm{OH}$ bend (H-bonded) & o.o.p & $900-950$ \\
\hline \multicolumn{4}{|c|}{ Carboxylate Group } \\
\hline$v_{\mathrm{a}}(\mathrm{OCO})$ & asym. OCO stretch & in-plane & $1500-1660$ \\
\hline$v_{\mathrm{s}}(\mathrm{OCO})$ & sym. OCO stretch & in-plane & $1320-1420$ \\
\hline \multicolumn{4}{|c|}{ Phenyl Group } \\
\hline$\gamma_{\mathrm{a}}(\mathrm{C}-\mathrm{H})$ & asym. $\mathrm{C}-\mathrm{H}$ bend & o.o.p & 900 \\
\hline$\gamma(\mathrm{C}-\mathrm{H})$ & $\mathrm{C}-\mathrm{H}$ bend & o.o.p & 740 \\
\hline
\end{tabular}

${ }^{a}$ Abbreviations: o.o.p $=$ out-of-plane; asym. = asymmetric; sym. $=$ symmetric.

IR-active modes from TMA adsorbed at electrified solid/liquid interfaces, ${ }^{63,64}$ from DFT calculations for TMA isolated in vacuum and solid-state IR spectra of 3D Cu-TMA polymers ${ }^{57}$ and of TMA and related benzene carboxylic acids. ${ }^{65,66}$ Additionally, vibrational data from carboxylic acids adsorbed on metal surfaces is also used..$^{6-10,63}$ In Table 1, we list and assign the main modes observed in these experiments. We note that the frequency of these modes can shift as a function of the coordination of the carboxylic and carboxylate groups. The vibrational frequencies from this set of data can be used to characterize the functional groups of the TMA molecules. Moreover, by using the dipole selection rule, which states that only vibrational modes having a dynamic dipole component perpendicular to the surface are IR active, we can determine the orientation of the adsorbed species.

The low-coverage regime is dominated by molecular interaction with the metal surface. In spectra $a-c$, the observation of out-of-plane modes at 743 and $940 \mathrm{~cm}^{-1}$ for $\gamma(\mathrm{C}-\mathrm{H})$ of the phenyl group and $\pi(\mathrm{OH})$ of the acid group not only indicates the presence of these groups but that they are orientated with their planes parallel to the surface. This interpretation is supported by our DFT calculations that predict frequencies at 

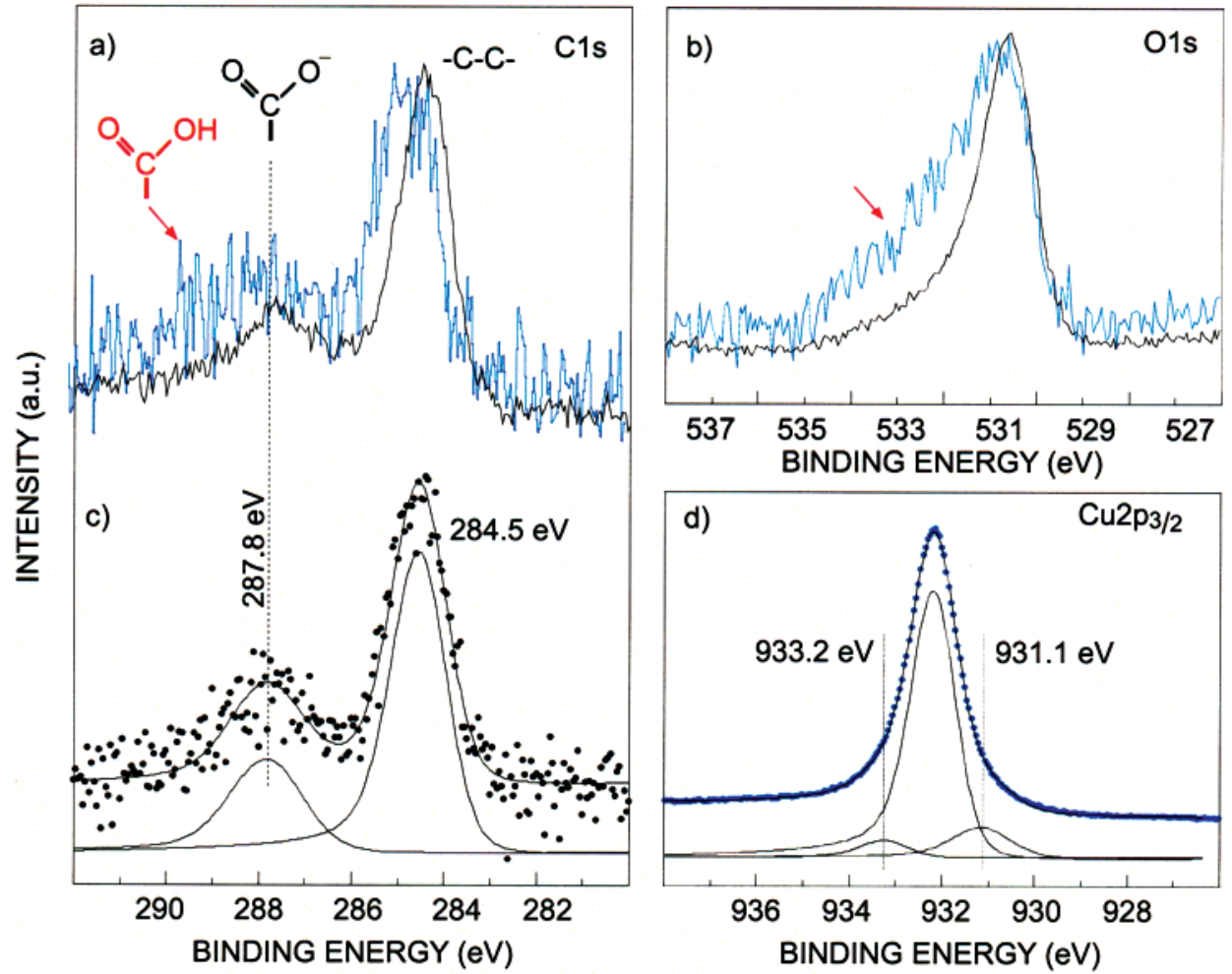

Figure 4. XPS spectra for low-coverage deposition of TMA on $\mathrm{Cu}(110)$; (a) $\mathrm{C} 1 \mathrm{~s}$ spectra and (b) O1s spectra after deposition at $100 \mathrm{~K}$ (blue curve) and after warming up to room temperature (black curve), (c) C1s spectrum after room-temperature deposition with line shape analysis, and (d) $\mathrm{Cu} 2 \mathrm{p}_{3 / 2}$ spectrum after $100 \mathrm{~K}$ deposition with line shape analysis. All spectra are normalized to the maximum peak intensity.

729 and $944 \mathrm{~cm}^{-1}$ whose eigenmodes involve atomic displacements orthogonal to the phenyl group, in agreement with the assignation described above. The other two prominent peaks at 1427 and $1643 \mathrm{~cm}^{-1}$ might, in principle, be attributed to shifted $\delta(\mathrm{COH})$ and $v(\mathrm{C}=\mathrm{O})$ acid modes of TMA molecules which are tilted away from the surface plane. In fact, a tilted geometry would make these in-plane modes visible and could lead to an interaction with the surface such that the vibrational frequencies of the interacting groups are shifted. However, due to the symmetry of the molecule, at least one unperturbed acid group would always be pointing away from the surface and hence give rise to "regular" $v(\mathrm{C}=\mathrm{O})$ IR absorption bands, which are not seen. The only alternative explanation for the 1427 and 1643 $\mathrm{cm}^{-1}$ bands is that they originate from the symmetric and asymmetric stretching modes of carboxylate groups.

The observation of these latter modes signifies two things; first, even at these extremely low substrate temperatures, there must be some deprotonation of the acid groups, although its extent cannot be established. Second, for both carboxylate modes to be observed and hence be dipole active, they must be (at least slightly) rotated out of the molecular plane.

The differences between spectra b, c, and d mark the transition to coverages above the monolayer, where $\mathrm{H}$ bonding dominates, as shown by the appearance of a very broad $\mathrm{OH}$ stretch around $3200 \mathrm{~cm}^{-1}$ (spectra $\mathrm{d}-\mathrm{g}$ ). The bands around 1270, 1430, and $1710 \mathrm{~cm}^{-1}$ are associated with acid groups free of surface interactions and indicate the presence of non-flat-lying molecules. These frequencies have counterparts in the DFTcalculated spectrum at 1300,1426 , and $1715 \mathrm{~cm}^{-1}$. Under these conditions, no preferential orientation can be deduced as the in-plane and out-of-plane modes do not show any significant intensity variation. This is not unusual at very low temperatures as condensation of molecules above the monolayer coverage leads to their random aggregation and thus to the formation of an inhomogeneous overlayer. This is further reflected in the IR spectra as a splitting of the bands associated with several functional groups (see spectrum g) due to the different local environments that these groups experience.

Coverage-dependent IR measurements were also measured at $240 \mathrm{~K}$. The resulting spectra (not shown) are very similar to those shown in Figure 3, indicating that, up to this temperature, no significant change is occurring in the chemical nature and the spatial orientation of the adsorbed molecular species.

C. XPS Measurements. Core level photoemission spectra measured in the high-resolution XPS system were used for a further chemical analysis of the molecular layer at low temperatures. Figure $4 \mathrm{a}$ shows the $\mathrm{C} 1 \mathrm{~s}$ core level of a low-coverage TMA film deposited on $\mathrm{Cu}(110)$ at about $100 \mathrm{~K}$ (blue curve, preparation conditions similar to those of Figure 2). The strong peak at about $285 \mathrm{eV}$ can be associated with the carbon atoms of the phenyl ring, while the broad feature at higher binding energy spans the range of contributions of both carboxylic and carboxylate groups. ${ }^{11-14}$ The corresponding integrated intensities are in a 2:1 ratio, which demonstrates that the molecular carbon frame stays intact upon adsorption. Due to the low TMA coverage and corresponding low signal intensity, a precise peak assignment can only be done based on the heated high-coverage data presented in section V. However, the width of the photoemission signal in the higher binding energy region suggests that both carboxylic and carboxylate groups have to be present on the surface. This indicates that a partial deprotonation of the carboxylic groups occurs even at low temperatures, therefore supporting the IR results.

The O1s emission is displayed in Figure $4 \mathrm{~b}$ for the cold sample (blue curve). It comprises a peak at about $531 \mathrm{eV}$ and a broad shoulder at higher binding energies (red arrow). Again, a precise peak analysis is hampered by the low signal intensities. However, according to earlier work, ${ }^{11-14}$ the broad shoulder can be assigned to hydroxyl $(-\mathrm{OH})$ and carbonyl $(=\mathrm{O})$ groups in hydrogen-bonded carboxylic moieties. On the other hand, the 

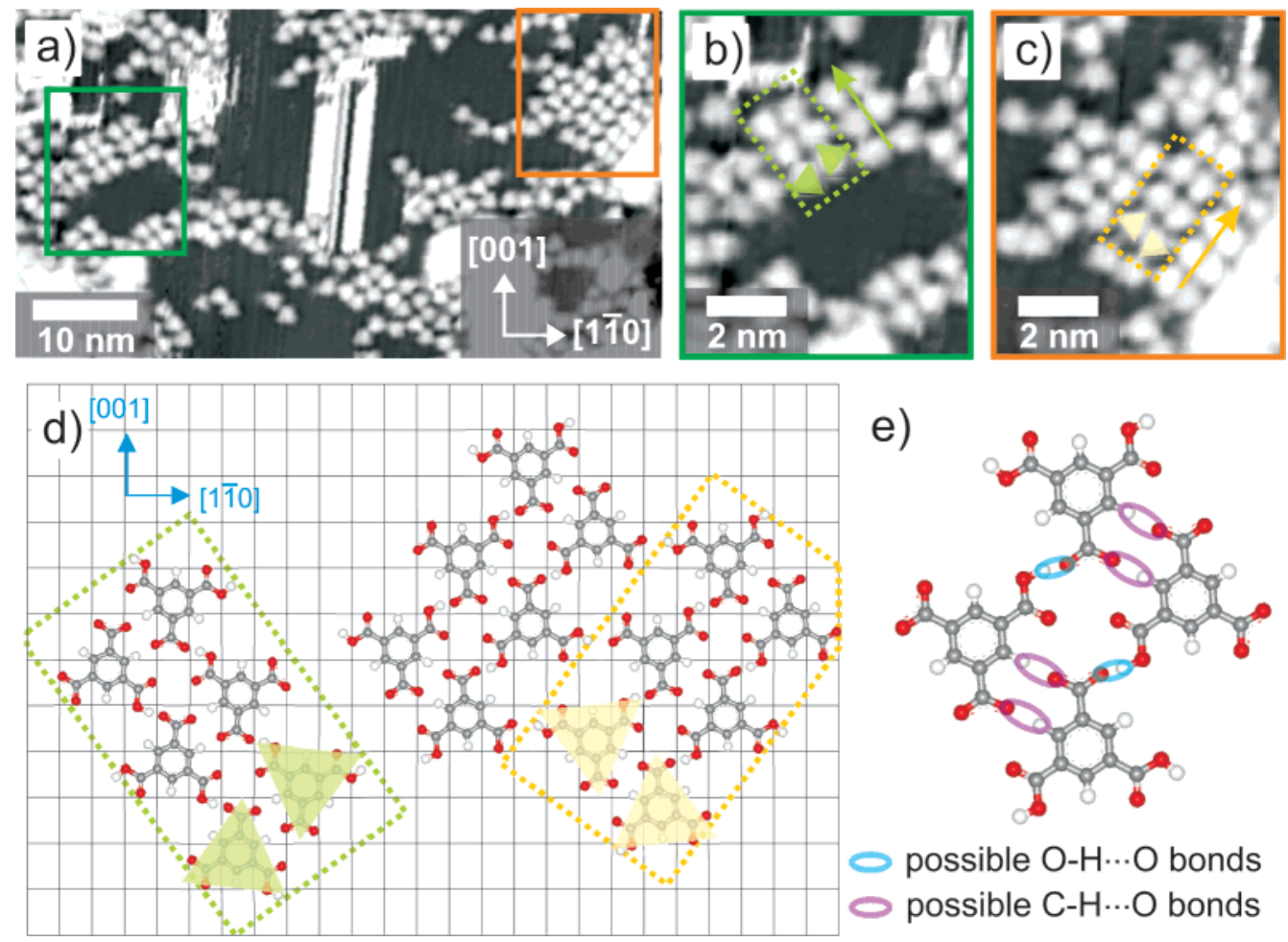

Figure 5. Adsorption of TMA on $\mathrm{Cu}(110)$ at $240 \mathrm{~K}$ (cooled to $40 \mathrm{~K}$ for measurement); (a) STM image showing formation of small chiral molecular networks; ( $b$ and c) magnified views of the regions marked in a. Two chiral molecular double rows are outlined by the dotted boxes. The corresponding main axes are indicated by arrows. (d) Model of the adsorption geometry for the two chiralities, and (e) enlarged view showing the positions where ionic hydrogen bonds can be formed.

peak at about $531 \mathrm{eV}$ is indicative of chemically identical oxygen atoms in carboxylate groups. ${ }^{11-13}$ As a consequence, the analysis of the oxygen energy region further confirms the simultaneous presence of intact and deprotonated carboxylic groups.

Figure $4 \mathrm{~d}$ shows the $\mathrm{Cu} 2 \mathrm{p}_{3 / 2}$ core level spectrum together with the results of a line shape analysis. At lower binding energies $(931.1 \mathrm{eV})$, a surface-shifted component can be seen that is already present on the clean surface. TMA deposition at $100 \mathrm{~K}$ leads to the evolution of an additional species at higher binding energy $(933.2 \mathrm{eV})$ that can be attributed to a $\mathrm{Cu}-\mathrm{O}$ environment. ${ }^{67}$ Heating to room temperature does not produce any significant variation in the copper spectra; within the experimental error, the line shape analysis results in the same components as those directly after deposition.

D. STM Measurements at $240 \mathrm{~K}$. STM measurements show that increasing the substrate temperature up to $210 \mathrm{~K}$ (during or after deposition) does not significantly affect the molecular organization. However, above $210 \mathrm{~K}$, there is some indication of the beginnings of a structural transformation (albeit involving only a few percent of the total TMA coverage). Such local changes resemble the carboxylate structures found at higher temperatures, which will be discussed in detail in section IV.

At substrate temperatures of $240-250 \mathrm{~K}$, a more pronounced molecular ordering is found (see Figure 5a), with the formation of locally ordered and densely packed islands which extend over several nanometers and incorporate some dozens of molecules. Since no significant variation can be observed in the corresponding IR spectra, the formation of molecular islands cannot be due to a different intermolecular interaction but must originate from a higher surface mobility of the TMA molecules induced by the higher substrate temperature.

The most frequently observed islands are composed of molecular double rows existing in two enantiomeric forms (see
Figure $5 \mathrm{~b}$ and c). The main axis of these double rows is rotated by $\pm\left(55 \pm 3^{\circ}\right)$ out of the [1 $\overline{1} 0$ ] direction, thus along [1 $\left.\overline{1} 2\right]$ and [112]. By comparing the orientation in islands of opposite chirality, it can be seen that the molecules inside of a domain are rotated by a small angle of $\pm\left(5 \pm 3^{\circ}\right)$ from an orientation fully aligned to the high-symmetry directions of the substrate. This rotation is clockwise and counterclockwise in Figure 5b and c, respectively.

Interestingly, extended honeycomb motifs of completely protonated TMA molecules similar to those formed for the deposition on $\mathrm{Ag}(001), \operatorname{Ag}(111),{ }^{12,14} \mathrm{Au}(111),{ }^{52} \mathrm{HOPG},{ }^{50,55} \mathrm{Ag} /$ $\mathrm{Si}(111),{ }^{54}$ and $\mathrm{Cu}(001)$ at low temperatures, ${ }^{24}$ are never observed on $\mathrm{Cu}(110)$. The most probable reason for this difference is the partial deprotonation of the TMA molecules as measured by IR and XPS for temperatures as low as $100 \mathrm{~K}$ (see sections III $\mathrm{B}$ and III C, respectively). A similar nonhexagonal arrangement of partially deprotonated TMA molecules has been recently reported by Payer et al. for the high-temperature deposition of TMA on $\operatorname{Ag}(111) .{ }^{14}$ There, it was theoretically demonstrated that supramolecular arrangements stabilized by ionic hydrogen bonds are energetically more favorable since they minimize the repulsive interactions of adjacent negatively charged oxygen atoms.

A tentative model of the $240 \mathrm{~K}$ adsorption structure on $\mathrm{Cu}$ (110) is shown in Figure 5d. The TMA molecules are partially deprotonated, in accordance with the IR and XPS measurements. The grid represents the substrate periodicity, and all of the TMA molecule fit for the STM measurements, as not only the molecular positions and orientations but also the small free $\mathrm{Cu}$ areas inside of the molecular islands are well reproduced. The molecules adopt four possible orientations resulting from the up and down position of the TMA tips with respect to the [110] direction and by the two aforementioned $\pm 5^{\circ}$ rotations. In a chiral domain (molecular double row), only molecules rotated 

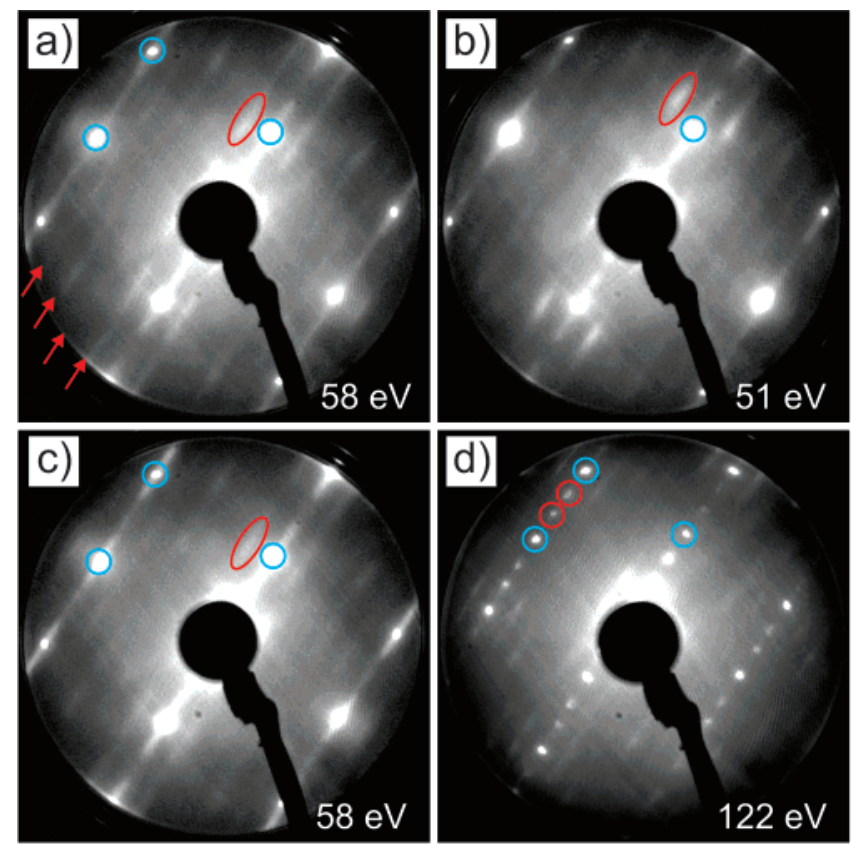

Figure 6. LEED patterns of low-coverage deposition of TMA on $\mathrm{Cu}$ (110); (a) $0.3 \mathrm{ML}$ deposited at room temperature. The $5 \times$ periodicity along the [110] direction is indicated by red arrows, and the substrate spots are indicated by blue circles (electron energy $58 \mathrm{eV}$ ). (b) The same film measured at an electron energy of $51 \mathrm{eV}$. The red ellipses in $\mathrm{a}$ and $\mathrm{b}$ demonstrate the energy-dependent shifting of the intensity along the one-fifth streaks. (c) $0.3 \mathrm{ML}$ deposited at room temperature and heated at $500 \mathrm{~K}$. (d) $0.5 \mathrm{ML}$ deposited at room temperature and heated at $500 \mathrm{~K}$. The $5 \times$ periodicity is still observed along [110] together with a $\times 3$ periodicity along [001], as indicated by the red circles.

in the same direction are found. Within such a double row, the supramolecular structure is optimized for multiple ionic hydrogen bonds (Figure $5 \mathrm{e}$ ). This results in a $\approx \pm 5^{\circ}$ rotation of the individual molecules and is the origin of the organizational chirality of the molecular islands. The connection between adjacent molecular double rows of the same chirality can also be ascribed to ionic hydrogen bonding. We note that the arrangement proposed in Figure $5 d$ is quite flexible since it can accommodate TMA molecules that are deprotonated in one or two positions. Moreover, a slightly different distribution of the carboxylic and carboxylate groups in partially deprotonated TMA molecules might be responsible for the various small homo- and heterochiral TMA-TMA structures observed in the upper and left part of Figure 5c.

Although the supramolecular structures formed at $240 \mathrm{~K}$ are locally ordered, the substrate temperature is evidently not sufficient for the development of a long-range periodicity. As a consequence, LEED measurements at this temperature present only the substrate $1 \times 1$ spots superimposed onto a diffused background (data not shown).

\section{Low Coverage at $300 \mathrm{~K}$ and Above: The Single Chain Phase}

A. LEED and XPS Measurements. In contrast to lowtemperature deposition (100-240 K) where no LEED superstructure evolves, the gradual appearance of long-range order can be detected at higher temperatures. Deposition of submonolayer coverages of TMA at $T \geq 280 \mathrm{~K}$ leads to the development of streaky diffraction lines at one-fifth-order positions in the direction of the long reciprocal unit vector (arrows in Figure $6 a$ ), which correspond to a $5 \times$ periodicity along the [1 $1 \overline{1} 0]$ crystal direction; no periodic order perpendicular to this is observed. Instead, the positions of intensity maxima within the five-fold streaks vary as a function of energy, which is typical for a disordered arrangement along [001] (cf. Figure $6 \mathrm{a}$ and $\mathrm{b}$ ). However, at higher coverage, a well-defined threefold periodicity does develop in the [001] direction, as depicted by the spots marked in Figure 6d (red circles), which corresponds to saturation of the low-coverage phase (see below).

The appearance of this structural order is accompanied by a substantial chemical change of the adsorbed molecules. The XPS measurements in Figure 4 show that both deposition and heating at room temperature result in an almost complete deprotonation of the carboxylic groups. As a consequence, the surface species becomes a 1,3,5-benzenetricarboxylate, referred to as trimesate (TM). After warming the molecular film to room temperature (black curve in Figure 4a), the C1s core level emission shows only the two carbon components, corresponding to the ring atoms at $284.5 \mathrm{eV}$ and to carboxylate species at $287.8 \mathrm{eV}$. Within the experimental error, no relevant emission intensity could be detected at binding energies around $289.4 \mathrm{eV}$, characteristic for intact carboxylic groups (see section $\mathrm{V} \mathrm{E}$ ). An almost identical spectrum is obtained after depositing a similar coverage directly at room temperature (Figure 4c).

The O1s spectra after room-temperature heating (black curve in Figure 4b) or deposition (not shown) provide a further indication for the deprotonation of the TMA molecules. In both cases, there is a strong reduction of the broad shoulder at around $533 \mathrm{eV}$ which is characteristic of intact carboxylic groups. The room-temperature spectra are dominated by the component at around $531 \mathrm{eV}$, which is typical of carboxylate moieties. ${ }^{11-13}$

Heating these low-coverage TM films to temperatures as high as $500 \mathrm{~K}$ does not significantly change either the LEED patterns (cf. Figure 6a and c) or the XPS spectra, indicating the absence of any further significant structural or chemical rearrangement.

B. STM Measurements. STM investigation of low-coverage molecular films deposited or heated at $T \geq 280 \mathrm{~K}$ reveals the formation of unidirectional molecular motifs. These have the form of chains running along the [1실 direction of the $\mathrm{Cu}$ (110) surface (see Figure 7). The chains consist of alternating bright triangular features (flat-lying TM molecules ${ }^{24-26,28,49,51,53}$ ) and faint round protrusions associated with $\mathrm{Cu}$ adatoms. ${ }^{24-26,28}$ The apparent height of the TM molecules of $1.40 \pm 0.30 \AA$ is nearly double the height of the $\mathrm{Cu}$ protrusion of $0.75 \pm 0.20 \AA$ when scanning at $-1 \mathrm{~V}$ and $1 \mathrm{nA}$. Faint protrusions at the end of the chains suggest that the chains are also terminated by $\mathrm{Cu}$ adatoms (see Figure 7b). One side of the TM triangle is always aligned to the [110] direction of the $\mathrm{Cu}$ substrate, implying that one functional group of the molecule points perpendicularly out of the chain while the other two are facing the $\mathrm{Cu}$ adatoms on each side. The whole structure can be described as a metalorganic coordination chain (MOCC). ${ }^{34}$

$\mathrm{The} \mathrm{Cu}$ atoms in these chains originate from the free copper adatoms that diffuse from the surface steps and kinks. Since their emission is the result of an activated process, their density depends very sensitively on the substrate temperature. ${ }^{7}$ This $2 \mathrm{D}$ adatom "gas" aids the deprotonation of the TMA molecules. In fact, although a partial deprotonation already occurs at low temperatures (see section III), the availability of these highly reactive free adatoms dramatically accelerates this process. ${ }^{12}$ The combination of these two effects explains the quite narrow temperature window that we observe for the transition to the MOCC structures. 


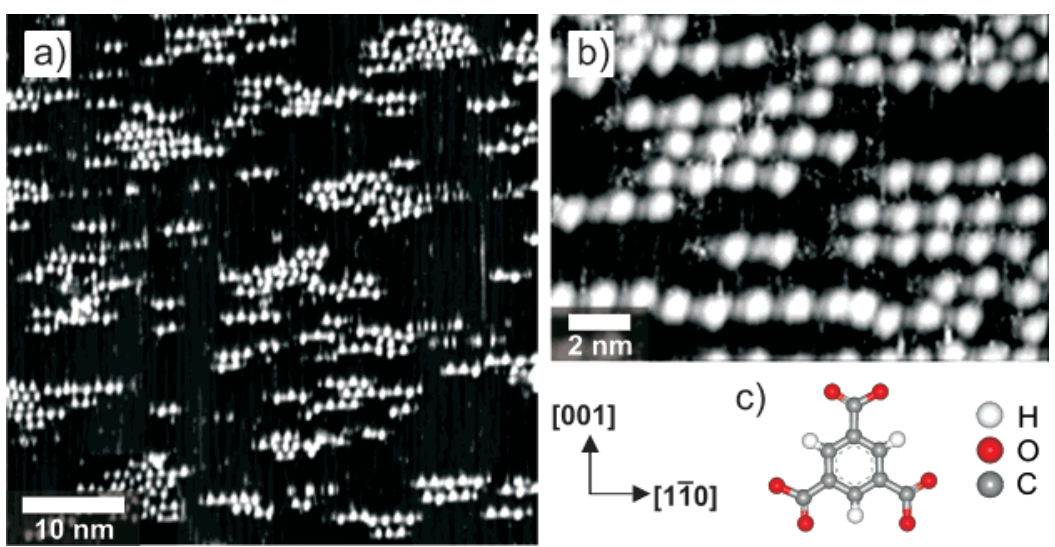

Figure 7. (a) Supramolecular structures created by TMA deposition at $240 \mathrm{~K}$ and subsequent heating to around $280 \mathrm{~K}$. (b) TMA deposition at 300 $\mathrm{K}$, magnified view. (c) Model of the TM molecule (deprotonated TMA).
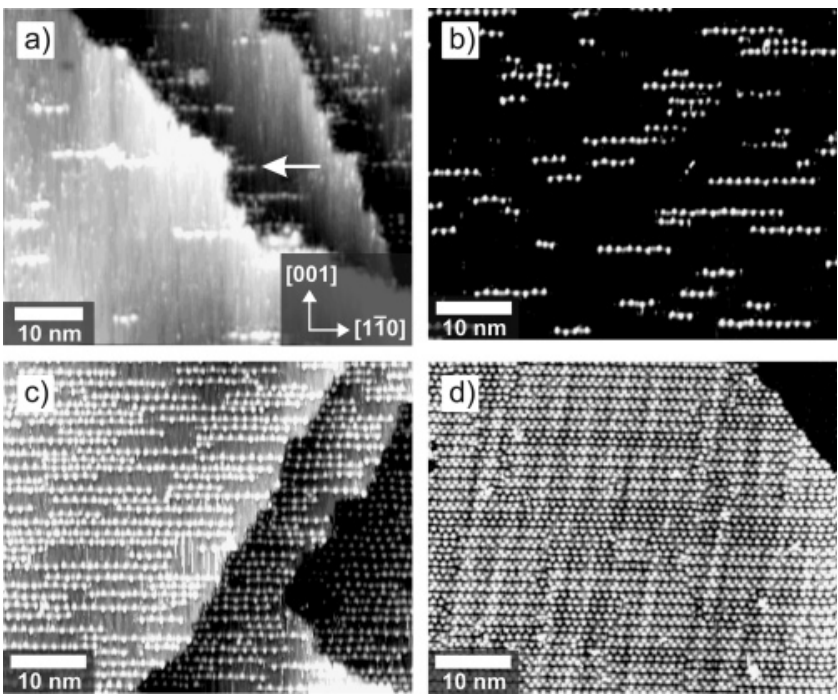

Figure 8. STM images of $\mathrm{TM} / \mathrm{Cu}(110)$, deposited at $300 \mathrm{~K}$ and heated to $(\mathrm{a}-\mathrm{c}) 380 \mathrm{~K}$ or (d) $500 \mathrm{~K}$. Coverage: (a) $0.04 \mathrm{ML}$, most of the molecules are attached at the lower side of the step edges (indicated by the arrow); (b) $0.10 \mathrm{ML}$, MOCC-S on terraces; (c) $0.36 \mathrm{ML}$, surface nearly covered with MOCC-S; (d) $0.9 \mathrm{ML}$, denser-packed structures comprising the MOCC-T and MOCC-D phases; see section V.

While for deposition or heating temperatures around room temperature the chains are typically kinked, a post-heating to $380 \mathrm{~K}$ or a deposition at this temperature creates highly ordered linear chains (see Figure 8). The local structure of the ordered chains is however the same as that for the chains grown at 300 $\mathrm{K}$, as also demonstrated by LEED (cf. Figure $6 \mathrm{a}$ and $\mathrm{c}$ ). For the chains grown at $280 \mathrm{~K}$, we observe an enhanced clustering tendency which is also present at $300 \mathrm{~K}$, although to a lesser extent. This may be due to intermolecular interactions mediated by some acid groups remaining protonated at the tips of the molecules and pointing out of the chains. Higher heating temperatures show no further change in the structure, even beyond $500 \mathrm{~K}$. The heated MOC chains will be referred to as MOCC-S (with "S" for single-chains) hereafter.

For low coverages, there is a preferential attachment of chains to step edges, with a TM molecule as the first link of the chain, showing the affinity for low-coordinated $\mathrm{Cu}$ atoms (see Figure 8a). With increasing coverage, the MOC chains begin to also nucleate on the terraces (see Figure $8 \mathrm{~b}$ ) and become longer and denser until they run all the way across the terraces (see Figure 8c). Above this limit, two densely packed phases are seen, the first being a transition phase (referred to as MOCC-T) and the second a double chain phase (MOCC-D; see Figure 8d), as will

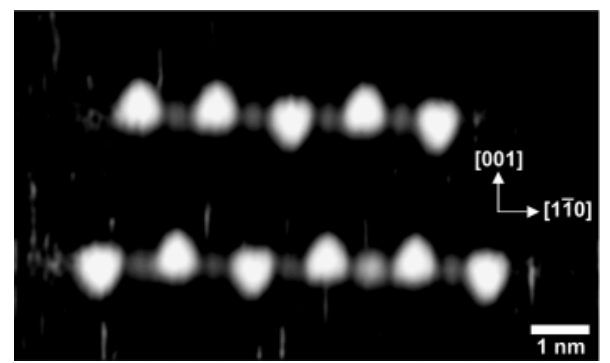

Figure 9. STM image of a $480 \mathrm{~K}$-heated MOCC-S structure of TM/ $\mathrm{Cu}(110)$.

be described later in section V. Only after the MOCC-D has completely covered the surface does the formation of a second layer start. As a consequence, we decided to normalize all coverage values to the coverage of a complete ideal MOCC-D phase (1 monolayer, ML) just before the nucleation of a second layer. Following this convention, the single chain MOCC-S phase spans the coverage range of $0-0.53 \mathrm{ML}$.

Figure 9 shows a high-resolution STM image of the MOCC-S phase. The periodicity along the chain is $12.69 \pm 0.15 \AA$, as measured from drift-free STM images, which corresponds to $4.96 \pm 0.06$, that is, five $\mathrm{Cu}$ lattice spacings in the [110] direction. This is in perfect agreement with the LEED measurements presented in section IV A. While the $5 \times$ periodicity is by far the most frequent (more than $77 \%$ of all cases), a periodicity of six or more $\mathrm{Cu}$ lattice spacings is sometimes found as a kind of dislocation along the chain (e.g., in the lower chain in Figure 9). In very few cases (3\% of the chain segments), a reduced periodicity of $4 \times$ is also found.

With increasing TM coverage, the chain density increases, but the chains always keep a minimum separation of three $\mathrm{Cu}$ lattice spacings along the [001] direction. The appearance of a well-defined $\times 3$ periodicity along [001] in the LEED measurement (Figure 6d) was indeed used as a reference point to calibrate the molecular deposition rate on the different experimental setups. Nevertheless, the MOCC-S phase cannot be described as a strict $(5 \times 3)$ superstructure since this would require a full correlation of TM positions between neighboring chains, including the directions in which they are pointing. On the contrary, we observe that, within a chain, the third functional group of each TM molecule (its "tip") points up or down without regularity. Table 2 lists a statistical evaluation of the absolute and mutual orientation of the TM tips.

As can clearly be seen, no preferential up or down orientation exists for the MOCC-S, as expected from the symmetry of the substrate. Moreover, neither parallel nor antiparallel alignment 


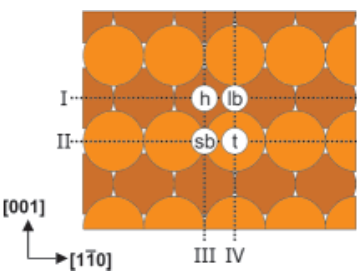

Figure 10. High-symmetry sites on $\mathrm{Cu}(110)$; h: hollow, lb: long bridge, sb: short bridge, and t: top. Lines I-IV: high-symmetry lines of the substrate. Line I identifies the closed-packed atomic troughs and line II the atomic rows.

TABLE 2: Statistical Analysis of the TM Orientation

\begin{tabular}{lccccc} 
& $\begin{array}{c}\text { coverage } \\
\text { (ML) }\end{array}$ & $\begin{array}{c}\% \\
\text { up }\end{array}$ & $\begin{array}{c}\% \\
\text { down }\end{array}$ & $\begin{array}{c}\% \\
\text { parallel }\end{array}$ & $\begin{array}{c}\% \\
\text { antiparallel }\end{array}$ \\
\hline MOCC-S & 0.08 & 55.6 & 44.4 & 52.7 & 47.3 \\
MOCC-S & 0.22 & 48.0 & 52.0 & 47.7 & 52.3 \\
MOCC-S & 0.38 & 48.8 & 51.2 & 55.8 & 44.2 \\
MOCC-S \& T & 0.64 & 48.6 & 51.4 & 71.1 & 28.9
\end{tabular}

is favored for neighboring TMs in a chain (see right-most columns of Table 2). A preferred parallel orientation exists though for the MOCC-T structures forming in the transition regime, which will be discussed in section $\mathrm{V} B$.

C. Determination of the MOCC-S Structure. The statistical analysis in Table 2 can be used to extract information about the adsorption geometry of the MOCC-S. Since no preferential up or down orientation and no preferential parallel or antiparallel configuration is found, the configurations must be degenerate in energy. As a consequence, we can safely assume that all TM molecules sit on equivalent substrate sites. Moreover, the position of a $\mathrm{Cu}$ protrusion in between two antiparallel TM molecules must be invariant for reflections with respect to planes parallel to (001). This implies that such a protrusion must lie along a mirror line parallel to the [1 $1 \overline{1} 0]$ direction of the substrate, that is, along line I or line II in Figure 10. Again, for mirrorsymmetry reasons, a $\mathrm{Cu}$ protrusion between two parallel TMs must be located either on line III or line IV in Figure 10. As a consequence, the $\mathrm{Cu}$ protrusions must be located on the intersections of these four lines (see definitions in Figure 10), while the centers of the TM molecules have to reside on highsymmetry lines parallel to the [001] direction (line III or line IV in Figure 10).

A direct experimental determination of the adsorption site can, in principle, be drawn from STM topographies that show a contemporary atomic resolution of the substrate and a molecular resolution of the chains. In practice this approach is limited as the STM measures local electronic densities and not the geometric topography. Depending on the electronic states of the tip and surface, atomic troughs may in fact be imaged as rows and vice versa. Seemingly "inverted" topographies due to specific electronic tip states have been reported for various substrates. ${ }^{68-70}$ As a consequence, we decided to resort to different measurements for determining the precise adsorption sites.

In order to establish the position of the chains relative to the [001] direction of $\mathrm{Cu}(110)$ (on atomic troughs or rows), STM manipulation experiments were performed on MOCC-S structures cooled to $100 \mathrm{~K}$. After measuring at normal scanning conditions (about $-1 \mathrm{~V}, 1 \mathrm{nA}$ ), the tunneling resistance was strongly decreased to $3 \mathrm{M} \Omega$ while moving the tip perpendicular to a chain (see line 1 in Figure 11a). By this, individual TM molecules were moved from the chain, which was confirmed by switching back to normal scanning conditions (see Figure $11 \mathrm{~b}$ and c). By iteratively removing all TM molecules from a
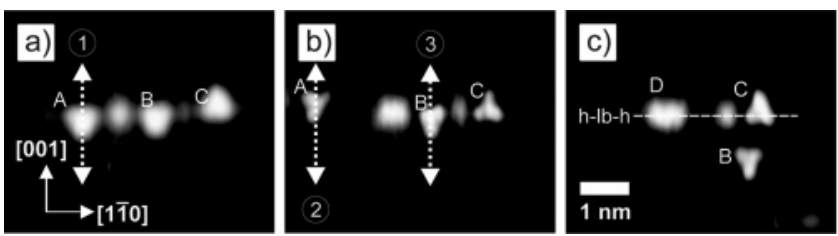

Figure 11. Example of low-temperature STM manipulation. (a) STM image of a chain of three TM molecules $\mathrm{A}, \mathrm{B}$, and $\mathrm{C}$ with an intermolecular distance of 6 and $5 \mathrm{Cu}$ lattice spacings, respectively. (b) Same area after low-resistance scanning along line 1; (c) after two further manipulations at positions 2 and 3. The new $\mathrm{Cu}$ island $\mathrm{D}$ identifies the atomic trough line. Changes in the molecular shape arise from tip switches. Molecule A has been moved out of the field of view.

chain, 1D $\mathrm{Cu}$ islands were created, which can naturally be expected to be located upon atomic troughs of the substrate. $\mathrm{Cu}$ adatoms within the chain were found to reside on the same line as these artificially created 1D island (line I in Figure 10). As a consequence, the position of the $\mathrm{Cu}$ protrusion within the MOCC-S chains was determined to be either hollow (h) or longbridge (lb).

For determining the position of $\mathrm{TM}$ and $\mathrm{Cu}$ within the chain, that is, along the [110] direction, the $\mathrm{Cu}(110)-(2 \times 1)-\mathrm{O}$ stripe pattern ${ }^{71-73}$ was employed as a reference. These stripes consists of chains of alternating $\mathrm{Cu}$ and oxygen atoms running along every second [001] row, that is, perpendicular to the [110] troughs. The deposition of TM in the wide $\mathrm{Cu}$ areas separating the stripes (data not shown) allowed us to deduce the TM and $\mathrm{Cu}$ protrusion sites in relation to the well-known $\mathrm{Cu}-\mathrm{O}$ structure. It should be noted that any influence of electronic inversion imaging is avoided by this approach as the periodicity of the stripes is double the periodicity of the $\mathrm{Cu}$ lattice along the [110] direction.

Combining both results, STM measurements find the TM to be located with its central phenyl ring close to the short-bridge site (sb) and the center of the $\mathrm{Cu}$ protrusion on the long-bridge site (lb). This result is in contradiction to a simplistic adsorption model of a chain of alternating TM molecules and $\mathrm{Cu}$ adatoms, $-\mathrm{TM}-\mathrm{Cu}-\mathrm{TM}-\mathrm{Cu}-\ldots$, for two reasons; first, $\mathrm{a} \mathrm{Cu}$ adatom on a lb instead of a $\mathrm{h}$ site is counterintuitive, and second, the distances between the oxygen atoms of TM and the $\mathrm{Cu}$ atom would be more than $2.8 \AA$, which is a relatively large value in comparison to the typical bond length of 1.9-2.2 $\AA$ reported in the literature. ${ }^{74}$ To resolve these issues, DFT calculations were used to determine the precise geometric structure of the chains.

D. DFT Analysis. Initially, all possible adsorption sites for the individual TM and $\mathrm{Cu}$ were compared. The results showed that the center of the phenyl ring favors the sb site and the $\mathrm{Cu}$ adatom favors the $\mathrm{h}$ site. The deprotonated TM is strongly bound to the surface, with a binding energy of $8.37 \mathrm{eV}$. It turns out that the adsorption process is forerun by the TMA dehydrogenation, which is catalyzed by the metal surface. Dehydrogenating TMA is indeed highly endothermic in vacuum [TMA $(\mathrm{g}) \rightarrow$ $\mathrm{TM}(\mathrm{g})+3 / 2 \mathrm{H}(\mathrm{g})-8.13 \mathrm{eV}]$, but at the surface, this is compensated by the adsorption of TM $(8.37 \mathrm{eV})$ and by the hydrogen dissociation leading to stable $\mathrm{H}$ adatoms $\left[3 / 2 \mathrm{H}_{2} \rightarrow\right.$ $3 \mathrm{H}(\mathrm{a})+0.42 \mathrm{eV}$ on clean $\left.\mathrm{Cu}(001)^{75}\right]$. The overall process (TMA deprotonation and adsorption) releases the energy $E_{\mathrm{TM}}^{\text {ads }}$ $=0.66 \mathrm{eV} /$ molecule

In order to compare the energetics of the different structures, it is convenient to introduce the formation energy per cell $F$

$$
F=E_{\mathrm{coh}}+N_{\mathrm{TM}} E_{\mathrm{TM}}^{\mathrm{ads}}-N_{\mathrm{Cu}} E_{\mathrm{Cu}}^{\mathrm{form}}
$$



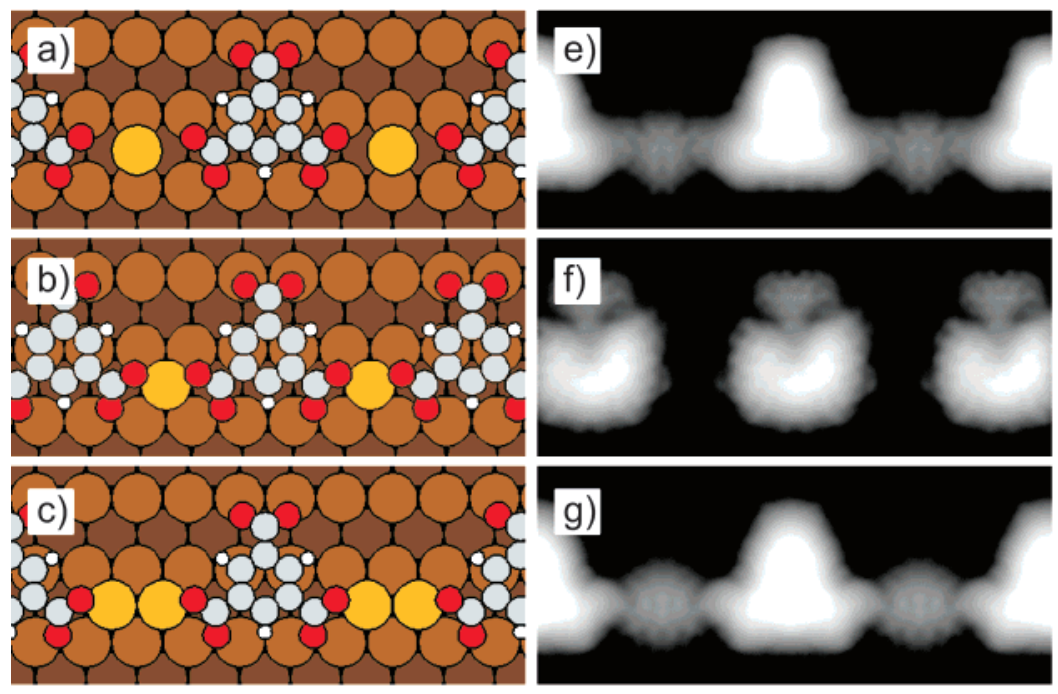

d)
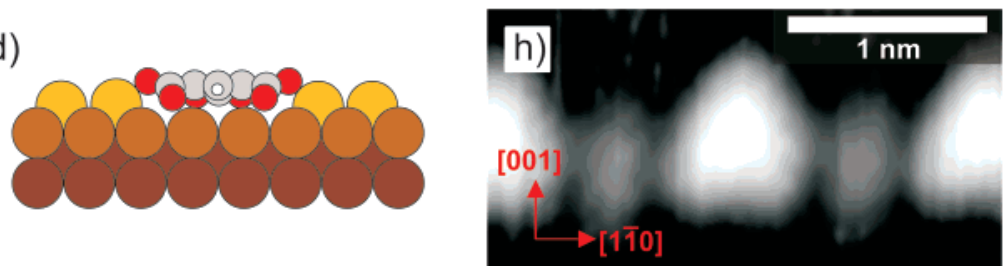

Figure 12. DFT results for different possible geometries of MOCC-S; $(a-c)$ fully relaxed atom configurations in top view, (d) side view of configuration $\mathrm{c},(\mathrm{e}-\mathrm{g})$ corresponding simulated STM images, and $(\mathrm{h})$ experimental image on the same scale. $(\mathrm{a}$ and e) $(5 \times 3)$ geometry with one adatom; $(\mathrm{b}$ and f) $(4 \times 3)$ configuration; and $(\mathrm{c}, \mathrm{d}$, and $\mathrm{g})(5 \times 3)$ configuration with a $\mathrm{Cu}$ dimer, lowest-energy structure.

TABLE 3: Energetics of the MOCC Structures ${ }^{a}$

\begin{tabular}{lcccc}
\hline \multicolumn{1}{c}{ structure } & $E_{\text {coh }}$ & $F$ & $F / N_{\mathrm{TM}}$ & $F / N_{1 \times 1}$ \\
\hline $5 \times 3$ MOCC-S & 1.30 & 1.09 & 1.09 & 0.073 \\
$4 \times 3$ & 0.64 & 0.86 & 0.86 & 0.072 \\
$5 \times 4$ & 1.83 & 1.41 & 0.71 & 0.071 \\
$4 \times 4$ MOCC-D & 1.09 & 1.55 & 0.77 & 0.097
\end{tabular}

${ }^{a}$ Variables: $E_{\mathrm{coh}}$, cohesive energy from the isolated adsorbed fragments; $F$, formation energy according to eq $1 ; F / N_{\mathrm{TM}}$, formation energy per molecule; $F / N_{1 \times 1}$, formation energy per number of substrate unit cells. All energies are in eV.

Here, $E_{\mathrm{coh}}$ is the cohesive energy gained by forming the chain from isolated fragments (TMs and adatoms), $E_{\mathrm{Cu}}^{\mathrm{form}}$ is the adatom formation energy $(0.44 \mathrm{eV})$, while $N_{\mathrm{TM}}$ and $N_{\mathrm{Cu}}$ are the number of molecules and adatoms involved in one cell of the structure, respectively.

For modeling the chain, a periodically repeating (-TM$\mathrm{Cu}-$ ) geometry was initially tried. When complexed with $\mathrm{Cu}$ adatoms, the energetically most favorable site for the TM is in a position between sb and h, closer to sb; Figure 12a. Although the simulated STM image does fit quite well with the experimental data (compare Figure $12 \mathrm{e}$ and $\mathrm{h}$ ), the $\mathrm{Cu}$ adatom is unstable in the $\mathrm{lb}$ site. In fact, the $\mathrm{Cu}$ atom would naturally relax into one of the neighboring $\mathrm{h}$ sites and thus be closer to one of the TMs, which lowers the total formation energy by $0.86 \mathrm{eV}$. As this would cause the chain to either break up or to relax into a $4 \times$ periodicity (Figure $12 b$ and $f$ ), both of which are not observed experimentally, we can rule this out as a possible structure.

In fact, the lowest-energy structure is formed by a periodic $(-\mathrm{TM}-\mathrm{Cu}-\mathrm{Cu}-$ ) geometry, where two $\mathrm{Cu}$ adatoms separate neighboring TMs (see Figure 12c). This structure, where the two $\mathrm{Cu}$ adatoms are in $\mathrm{h}$ sites and the TM is positioned between $\mathrm{sb}$ and $\mathrm{h}$ (closer to $\mathrm{sb})$, is the lowest-energy configuration ( $E_{\mathrm{coh}}$ $=1.30 \mathrm{eV}$ ) and has $5 \times$ periodicity (Table 3 ). Its formation energy $F=1.09 \mathrm{eV}$ is $0.23 \mathrm{eV}$ larger than that of a configuration having $4 \times$ periodicity $(0.86 \mathrm{eV})$. We notice that the formation energy per unit cell of the substrate, $F / N_{1 \times 1}$, is very similar for the two structures $(0.073 \mathrm{eV}$ for $5 \times$ MOCC-S and $0.072 \mathrm{eV}$ for $4 \times$ periodicity), suggesting that the system could switch to a $4 \times$ periodicity at high TM coverage. Indeed, as it will be shown in the following, the preferred structure at these conditions has $4 \times$ periodicity, although its geometry is different from the one depicted in Figure 12b.

This structure gives a more realistic $\mathrm{Cu}$-carboxylate bond length of $2.02 \AA$, in agreement with the values reported for $\mathrm{Cu}$ oxides. ${ }^{74}$ Furthermore, as can be seen from Figure $12 \mathrm{~g}$, the simulated STM image of this structure shows a single spot for the two $\mathrm{Cu}$ adatoms, which is in perfect agreement with the STM observation (Figure $12 \mathrm{~h}$ ). Additionally, the apparent heights obtained from the simulated STM image of $1.70 \AA$ for TM and $0.90 \AA$ for $\mathrm{Cu}$ are in good agreement with the STM measurements of $1.40 \pm 0.30$ and $0.75 \pm 0.20 \AA$, respectively.

The fact that a $\mathrm{Cu}$ dimer appears as a single spot, whereas a single $\mathrm{Cu}$ adatom in a hollow site does not produce any significant signature (both in simulated and experimental STM images; see Figure 12), suggests that adatoms may be incorporated into surface-supported metal-organic structures without being "visible" in STM. This may be applicable to other systems where structural models derived from experimental data gave poor fits, particularly for supramolecular systems involving carboxylic or carboxylate groups.

The DFT analysis of this periodic $(-\mathrm{TM}-\mathrm{Cu}-\mathrm{Cu}-)$ structure also shows that the TM is located $1.14 \AA$ above the outermost $\mathrm{Cu}$ layer. The molecule binds to the substrate mostly via the unsaturated carboxylate groups, which bend toward the surface by $9^{\circ}$ (those bound to the $\mathrm{Cu}$ adatom) and $15^{\circ}$ (those bound to the surface) and twist toward the $\mathrm{Cu}$ adatoms by $20^{\circ}$ with respect to the plane of the phenyl ring (Figure $12 \mathrm{~d}$ ). As a result, one of the carboxylate $\mathrm{O}$ atoms binds with the $\mathrm{Cu}$ adatom, and the other binds to the surface. In this geometry, the carboxylate groups are twisted and bent out of the molecular 


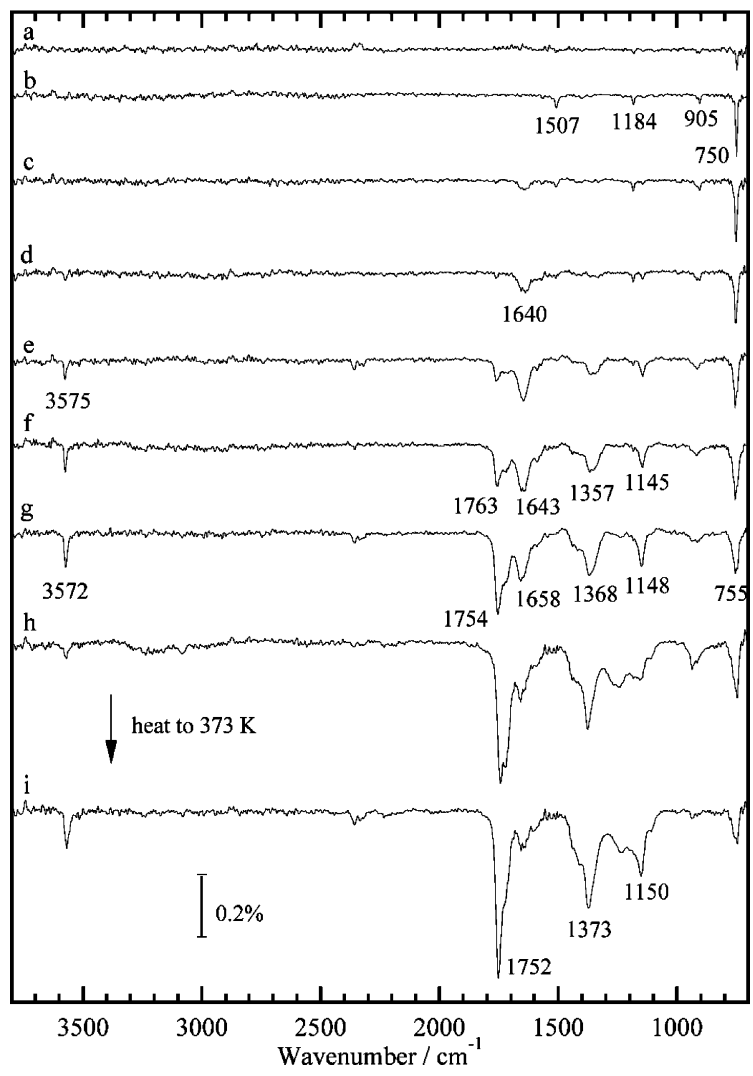

Figure 13. IR spectra measured as a function of increasing TMA coverage at $300 \mathrm{~K}$. The MOCC-S phase is correlated with spectra a-c. Spectrum $\mathrm{h}$ corresponds to the saturated layer as it does not substantially change with increased exposure.

plane so that the dipole moments associated with the $v_{\mathrm{a}}(\mathrm{OCO})$ and $v_{\mathrm{s}}(\mathrm{OCO})$ vibration modes have a component perpendicular to the substrate. This is most probably the reason why, although the overall orientation of the TM molecules is parallel to $\mathrm{Cu}$ (110), a signature of the carboxylate groups is still visible in the IR spectra of deprotonated TMA molecules complexed with $\mathrm{Cu}$ adatoms (section IV E).

E. IR Measurements. The coverage-dependent IR spectra measured at $300 \mathrm{~K}$ in Figure 13 provide evidence supporting the XPS, STM, and DFT results. Spectra $\mathrm{a}-\mathrm{c}$ correspond to the low-coverage phase $(<0.53 \mathrm{ML}$ with LEED patterns similar to those in Figure $6 \mathrm{a}$ and d), where the main bands are at 750 , 905,1184 , and $1507 \mathrm{~cm}^{-1}$. At higher coverage, the spectra change with the appearance of additional bands, which will be discussed in section $\mathrm{V}$ A. However, for low coverage, the strongest band at $750 \mathrm{~cm}^{-1}$ is again the out-of-plane $\mathrm{C}-\mathrm{H}$ bend of the phenyl ring, confirming the flat-lying orientation of the molecules. The band at $905 \mathrm{~cm}^{-1}$ could be a shifted out-ofplane $\mathrm{O}-\mathrm{H}$ bend, which was found at $940 \mathrm{~cm}^{-1}$ at lower temperatures (see Figure 3). However, since deprotonation of the carboxylic groups is shown by XPS and hydrogen-bonding motifs are not observed in STM, this band is most probably due to asymmetric out-of-plane $\mathrm{C}-\mathrm{H}$ bending modes which also exist in this frequency region (see Table 1). The two bands observed at higher frequency are in the range of in-plane modes, although they are not at the frequency of the acid groups, which are seen at higher coverages. Using the structural results of DFT, the higher frequency band at $1507 \mathrm{~cm}^{-1}$ can be assigned to the asymmetric stretch of a carboxylate group. Generally, the frequency and intensity of this latter band depends on the coordination of the carboxylate group. In this case, the weak intensity indicates that the carboxylate group is only slightly rotated out of the molecular plane (as seen from Figure 12d), while the frequency indicates that the group is symmetrically bound with two similar $\mathrm{C}-\mathrm{O}$ bond lengths. ${ }^{76}$ The IR spectra of the MOCC-S phase do not change upon heating to $380 \mathrm{~K}$, which indicates that the improvement in the structural order seen in the STM is not due to a chemical change or reorientation of the molecules.

\section{High Coverage at High Temperature: The Double Chain Phases}

A. IR Measurements. As already anticipated in section IV $\mathrm{B}$, the structure of the MOC chains changes when the molecular coverage exceeds $0.53 \mathrm{ML}$. We begin the analysis of the highcoverage phases by interpreting the IR data shown in spectra $\mathrm{d}-\mathrm{j}$ in Figure 13. The transition into the high-coverage phase starts with the attenuation of the band at $1507 \mathrm{~cm}^{-1}$ and the concomitant appearance of a band at $1640 \mathrm{~cm}^{-1}$ ( spectra $\mathrm{c}-\mathrm{e}$ ). This latter mode maximizes in intensity and remains relatively constant thereafter. In accordance with the low-temperature data (see section III B), this mode is also assigned to the asymmetric carboxylate stretch of molecules which are at least partially oriented perpendicularly to the surface. The large shift in frequency with respect to the lower-coverage data is related to a change in the coordination of the carboxylate group from being symmetrically to being asymmetrically bound. Therefore, the IR data suggest that at $300 \mathrm{~K}$, a partial reorientation of the molecules takes place when the coverage exceeds the full MOCC-S phase. This is presumably caused by compression of the adlayer. However, the absence of a significant intensity attenuation of the main out-of-plane mode at $750 \mathrm{~cm}^{-1}$ indicates that this phenomenon is much less pronounced than that for the adsorption of benzoic and terephthalic acids on $\mathrm{Cu}(110){ }^{6-8,10}$

As the coverage increases further (spectra $\mathrm{e}-\mathrm{g}$ ), additional bands which are associated with the acid groups appear at 3575 , 1763,1357 , and $1145 \mathrm{~cm}^{-1}$. With the dosing flux used in this study, spectrum $\mathrm{h}$ represents the saturated layer since it does not significantly change with increased exposure and thus cannot be ascribed to a multilayer. The appearance of in-plane acid group modes indicates that the extra adsorbed molecules do not have their molecular planes parallel to the surface but are most likely bound upright. These molecules may either be adsorbed on the remaining free areas of the $\mathrm{Cu}$ substrate (remained free because of defects in the MOCC-S structure; see Figure 8) or even on the $\mathrm{Cu}$ adatoms bridging the flat-lying TM molecules. In both cases, this is a strong type of binding, as confirmed by heating experiments at $373 \mathrm{~K}$, which show only small changes in the IR spectra. At intermediate coverages, a sharp $(\mathrm{OH})$ stretch at $3575 \mathrm{~cm}^{-1}$ indicates the presence of acid groups free from $\mathrm{H}$ bonding, although the attenuation of this band with increasing exposure indicates that these upright molecules can form $\mathrm{H}$ bonds with their neighbors. LEED measurements performed on the saturated film at $300 \mathrm{~K}$ do not show any feature of a regular overlayer signature but only a very high background intensity with hardly visible substrate spots, indicating a high degree of disorder.

A quite different situation is observed when TMA molecules are directly adsorbed at $470 \mathrm{~K}$, Figure 14 . Also in this case, no second layer is formed, as demonstrated by the IR signal which, after having reached saturation (spectrum $\mathrm{f}$ ), remains unchanged regardless of TMA exposure. However, the spectra in Figure 14 are characterized by a notable near-absence of the modes associated with acid groups (only a weak $\mathrm{C}=\mathrm{O}$ stretch band at $1760 \mathrm{~cm}^{-1}$ is seen at saturation, spectrum $\mathrm{f}$ ), implying that these molecular films are composed of completely deprotonated TMA molecules. 


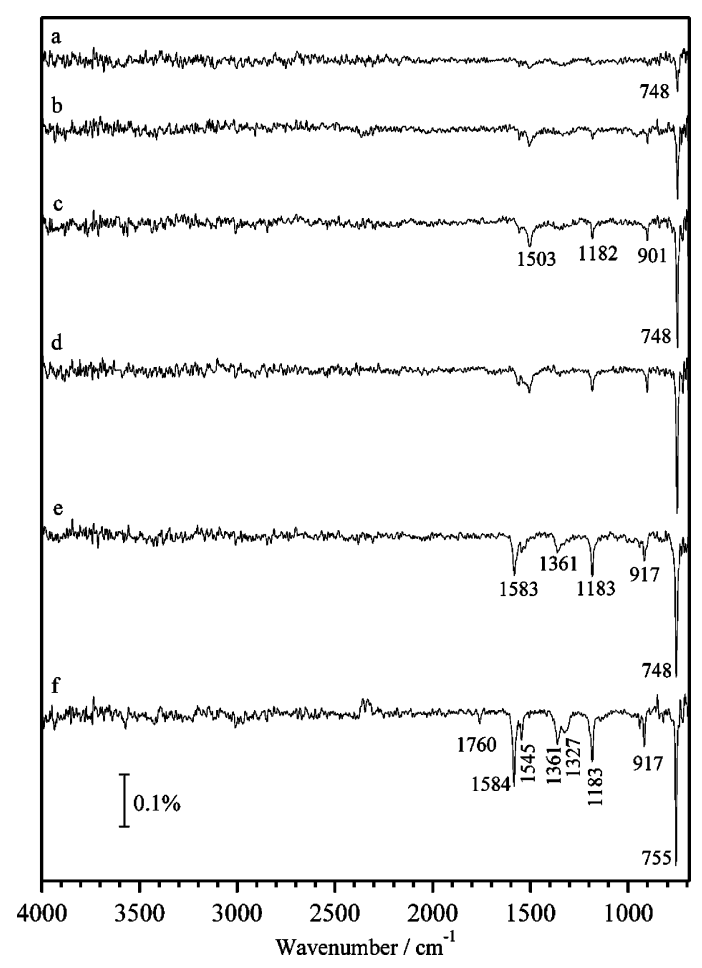

Figure 14. IR spectra measured as a function of increasing coverage at $470 \mathrm{~K}$. Spectrum $\mathrm{f}$ corresponds to a saturated monolayer.

The absorption peaks at low coverage (spectra $\mathrm{a}-\mathrm{d}$ ) are almost identical to the $300 \mathrm{~K}$ case (spectra a-c in Figure 13), indicating that the same MOCC-S low-coverage phase is formed. The higher-coverage phase (spectra e and f) presents, however, distinct differences with respect to the room-temperature data. An increased intensity is measured for the out-of-plane mode at $755 \mathrm{~cm}^{-1}$, indicating that at higher coverages, the majority of the molecules remain lying flat on the surface. More interestingly, the asymmetric carboxylate stretch mode appears at lower frequency, $1583 \mathrm{~cm}^{-1}$, indicative of a change in the bonding character of this group. This is supported by the DFT analysis in section $\mathrm{V} \mathrm{C}$ that indeed shows a difference in the $\mathrm{Cu}-\mathrm{O}$ binding between the low- (MOCC-S) and high-coverage phases (MOCC-D; see section V C). Another feature of the higher-coverage spectra is the appearance of modes around 1360 $\mathrm{cm}^{-1}$ that are very similar to what was found for the inorganic $\mathrm{Cu}-\mathrm{TM}$ polymer forming $2 \mathrm{D}$ sheets in $3 \mathrm{D}$ supramolecular crystals. ${ }^{57}$

Finally, we note that the IR absorption bands in these hightemperature spectra are typically much narrower than the corresponding low-temperature ones, which is often associated with improved ordering at the surface. This is also reflected in relatively sharp overlayer spots in LEED, which, for the saturated layer, shows the $(4 \times 4)$ structure shown in Figure $16 \mathrm{~b}$.

B. STM Measurements. In accordance with the LEED measurements, above a coverage of $0.53 \mathrm{ML}$ at $300 \mathrm{~K}$, no ordered structures are found in STM either. Partial ordering is observed to start only for heating temperatures of $380 \mathrm{~K}$ or higher, Figure 15a. A similar result can be obtained also without heating by leaving the surface at $300 \mathrm{~K}$ for about a day. In both cases, a few poorly resolved structures are still found in STM with an apparent height of at least $2.2 \AA$. In accordance with the IR measurements (section V A), these can be assigned to upright standing molecules bound either directly to the substrate or to adatoms of the first layer (see blue box in Figure 15a). The tips of the flat lying molecules within a chain are all aligned in the same direction (see Figure 15a and the last row in Table 2), and the tips of molecules in neighboring chains face each other, which maximizes their packing density. The periodicity in the direction perpendicular to the chains is thus increased from $\times 3$ to $\times 5$ substrate lattice spacings, leading to a higher molecular density, as now two chains are included in each cell. The in-chain periodicity is found to be either $4 \times$ or $5 \times$, creating locally ordered $(5 \times 5)$ or $(4 \times 5)$ structures (referred to as transition phase, MOCC-T). In addition, small domains with a $(4 \times 4)$ geometry are also found (MOCC-D; see section V C). The upright standing molecules described above are principally found at domain boundaries.

The transition between the various molecular configurations is more clearly seen for substrate temperatures of $500 \mathrm{~K}$. The higher surface mobility leads to the formation of large extended domains with few domain boundaries, in which upright molecules are rarely seen. For coverages between 0.53 and $0.6 \mathrm{ML}$, the MOCC-S and MOCC-T structures coexist. The latter increase with coverage and are exclusively observed between 0.6 and $0.8 \mathrm{ML}$, Figure 15b. A further coverage increase creates the $(4 \times 4)$ MOCC-D domains, which coexist with the $(4 \times 5)$ MOCC-T. The MOCC-D domains grow further with coverage, and at $1 \mathrm{ML}$, they cover the entire surface. At $500 \mathrm{~K}$, no additional molecules are incorporated into the saturated monolayer, and no second or higher layers are formed. Independent of the deposition temperature, a similar MOCC-D saturated monolayer can also be formed upon heating a substrate with a high molecular coverage to temperatures higher than $470 \mathrm{~K}$.

C. The Structure of MOCC-D. The structure of the MOC-D phase (see Figure 16a) is significantly different from that of the MOCC-S. The in-chain periodicity is reduced from $5 \times$ to $4 \times(3.96 \pm 0.15$ lattice spacings along [110]), while the periodicity perpendicular to the double chains is equivalent to four lattice spacings along [001]. The corresponding symmetry can also be seen from the LEED image in Figure 16b, which was taken after heating to $470 \mathrm{~K}$. The packing is substantially enhanced in comparison to that of the MOCC-S phase since the unit cell now comprises two TM molecules while having a similar extension (0.067 TM per substrate unit cell in MOCC-S and $0.125 \mathrm{TM}$ per substrate unit cell in MOCC-D). The reduced in-chain periodicity implies that only one $\mathrm{Cu}$ adatom resides between the molecules.

D. DFT Analysis. On the basis of the results obtained for the $(5 \times 3)$ structures (section IV D) and the high-resolution STM images in Figure 16e, the $(4 \times 4)$ structure is modeled by means of DFT. In comparison to the MOCC-S structure, the lowest-energy configuration (Figure 16c) is now characterized by having both the TM and the $\mathrm{Cu}$ adatoms in $\mathrm{h}$ positions. The simulated STM image agrees well with the experimental one, as can be seen by comparing Figure 16d and e.

From the fact that the higher-packed phases are never observed for coverages $<0.53 \mathrm{ML}$, we can conclude that the MOCC-S must be energetically more stable, as long as there is enough free substrate space for their formation. On the other hand, as soon as the full coverage in the MOCC-S phase has been exceeded, the system locally switches its configuration into a higher density phase in order to avoid the addition of molecules in an energetically unfavorable second layer. From a thermodynamic point of view, the gradual transition from the MOCC-S to the MOCC-D structures can be rationalized by analyzing the corresponding energetics. Two different mechanisms can lead to an increase in the density of TM molecules adsorbed on the metal surface. First, the reduction of the periodicity along the chain from $5 \times$ to $4 \times$, and second, the 


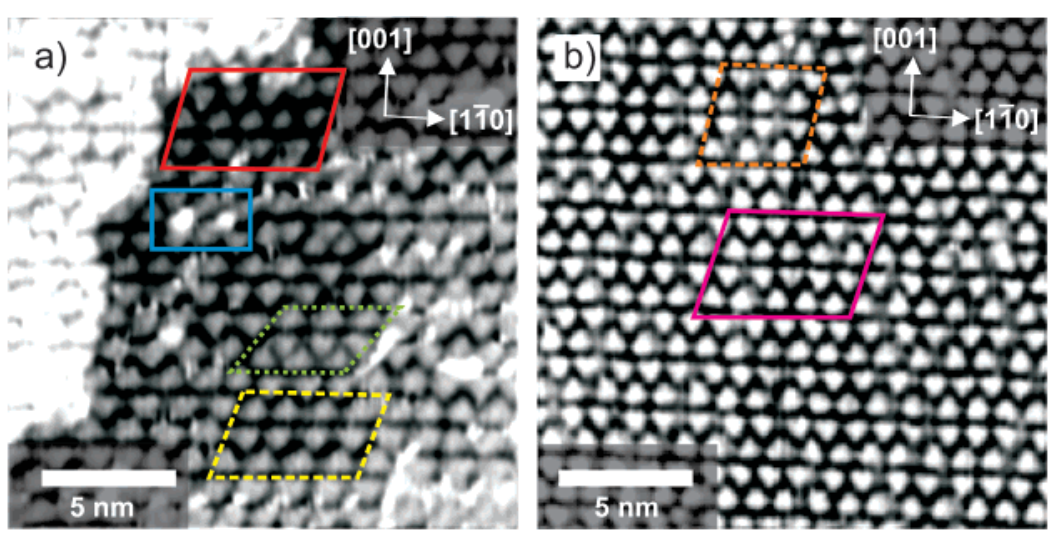

Figure 15. Intermediate TM coverage of 0.64 ML. (a) Heated to $380 \mathrm{~K}$; coexistence of MOCC-S (red solid), MOCC-T (yellow dashed), MOCC-D (green dotted), and interstitial impurities (blue solid). (b) Heated to $500 \mathrm{~K}$; MOCC-T in $(5 \times 5)$ domains (orange dashed) and $(4 \times 5)$ domains (magenta solid).
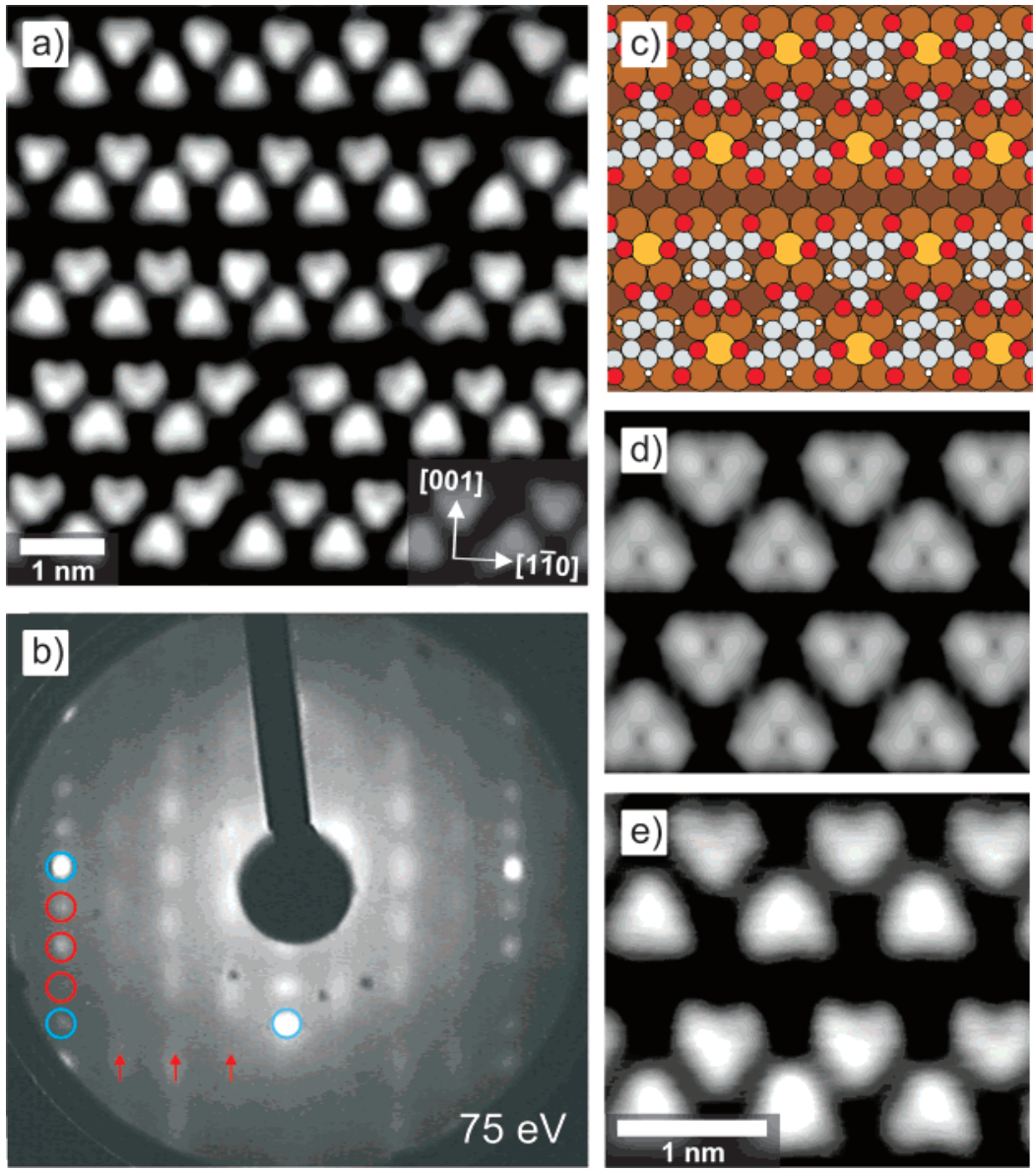

Figure 16. (a) Representative STM image of the MOCC-D phase. (b) LEED pattern showing a $(4 \times 4)$ structure. Blue circles indicate the substrate spots, and red circles and arrows represent the $(4 \times 4)$ periodicity. $(\mathrm{c}-\mathrm{e})$ Comparison of $(\mathrm{c})$ the fully relaxed lowest-energy adhesion structure, $(\mathrm{d})$ the simulated STM image as obtained from DFT, and (e) an experimental high-resolution STM image.

interlocking of neighboring chains. Both mechanisms are exploited in the formation of MOCC-D structures. As a consequence, in Table 3, we have compared the cohesive and formation energies for MOCC-S and MOCC-D with those for ideal $(4 \times 3)$ (reduced periodicity) and $(5 \times 4)$ structures (two interlocked TM per cell).

Being characterized by different numbers of molecules and adatoms per cell, the phase stability at low coverage of these structures is determined by their energy of formation per molecule $\left(F / N_{\mathrm{TM}}\right)$. According to Table 3 , the MOCC-S phase is the most stable one, in perfect agreement with the experimental observations for coverages $<0.53 \mathrm{ML}$. However, at higher coverages, when space is limited, the stability is governed by the formation energy per unit cell of the substrate $F / N_{1 \times 1}$. Note that reducing the periodicity to $(4 \times 3)$, or interlocking the chains, does not result, by itself, in an increase of $F / N_{1 \times 1}$. Only the combination of these two mechanisms is found to significantly stabilize the MOCC-D structure, increasing its $F / N_{1 \times 1}$ by $33 \%$ with respect that of the MOCC-S. This is due to formation of intermolecular $\mathrm{C}-\mathrm{H} \cdots \mathrm{O}$ ionic hydrogen bonds between the phenyl rings and the carboxylate groups of adjacent TM molecules (Figure 16c). The resulting $\mathrm{C}-\mathrm{H} \cdots \mathrm{O}$ distance is $3.2 \AA$ and is compatible with that reported in the literature for other 2D and 3D H-bonded structures. ${ }^{77,78}$ Moreover, we notice that the higher packing density increases both the bending (11.9 and $19.7^{\circ}$ for the groups bound to a $\mathrm{Cu}$ adatom and to 

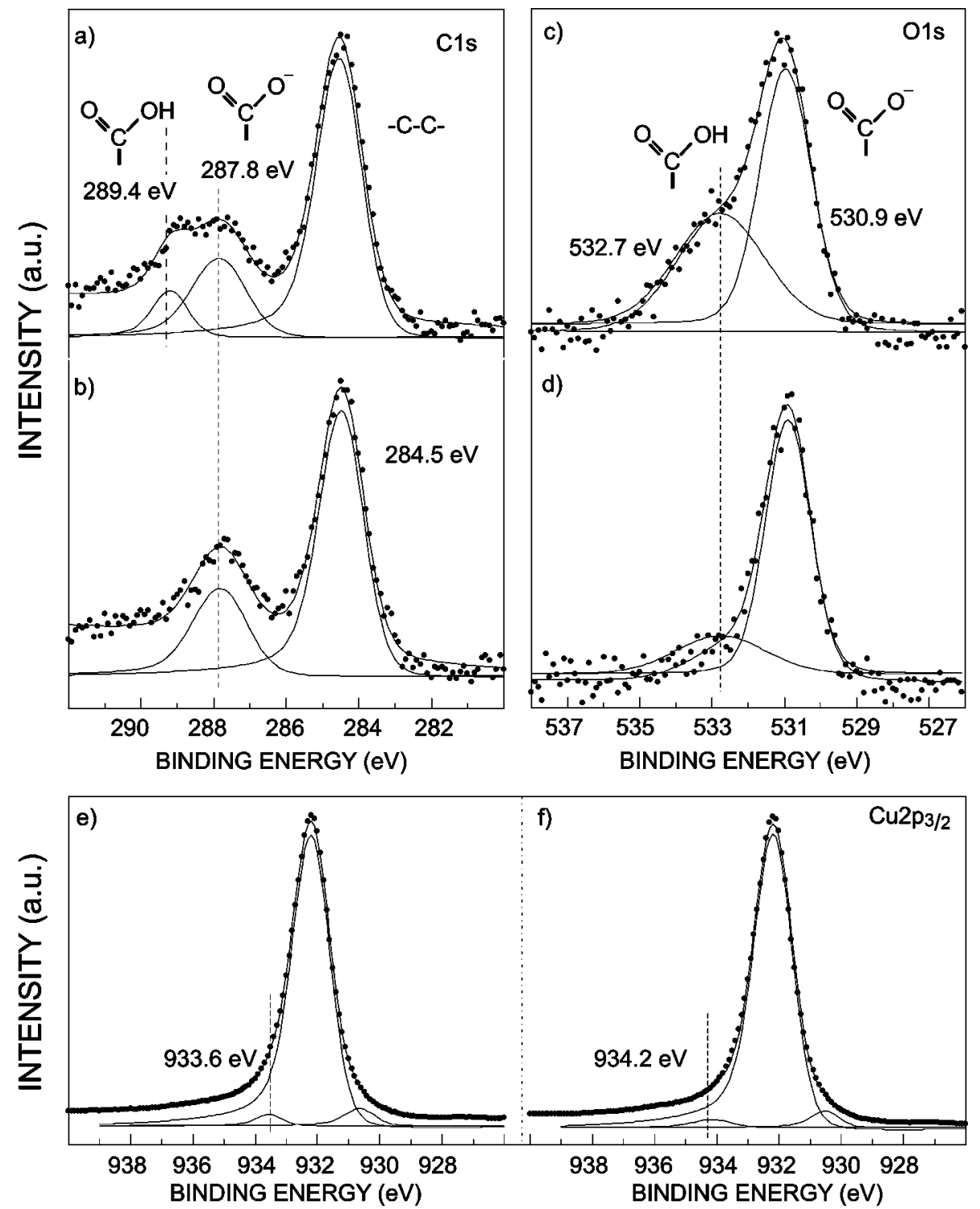

Figure 17. XPS spectra for high-coverage deposition (ca. $1 \mathrm{ML}$ ) of TMA on $\mathrm{Cu}(110)$ with fitted components indicated. (a and b) C1s spectra, (c and d) $\mathrm{O} 1 \mathrm{~s}$ spectra, and (e and f) $\mathrm{Cu} 2 \mathrm{p}_{3 / 2}$ spectra; a, c, and e are after room-temperature deposition, and b, $\mathrm{d}$, and $\mathrm{f}$ are upon heating at $500 \mathrm{~K}$. All spectra are normalized to the maximum peak intensity.

the surface, respectively) and twisting $\left(25^{\circ}\right)$ angles of the unsaturated carboxylate groups with respect to corresponding values in the MOCC-S structure.

We can conclude that, at low coverages, the phase stability of the MOCC-S single chains is determined by their highest energy of formation per molecule, while at high coverages, it is more convenient to form the MOCC-D phase rather than simply interlocking MOCC-S chains because of the higher number of $\mathrm{H}$ bonds in MOCC-D.

It should be noted that the completion of all of these phase transformations requires the displacement of several molecules and adatoms per unit cell. These movements are associated with certain kinetic barriers which manifest themselves in the heating and/or the long relaxation time necessary for these transitions to occur.

E. XPS Measurements. Figure 17 shows core level spectra for room-temperature deposition followed by heating to $500 \mathrm{~K}$ of about 1 ML TMA on $\mathrm{Cu}(110)$. The carbon frame of the molecule again stays intact, as seen from the $\mathrm{C} 1 \mathrm{~s}$ spectra. However, after deposition at $300 \mathrm{~K}$, two contributions at higher binding energies (289.4 and $287.8 \mathrm{eV}$ ) are present, indicating both carboxylic and carboxylate groups, respectively (Figure 17a). Similarly, the O1s spectra in Figure 17c show the carboxylate peak at $530.9 \mathrm{eV}$ together with a broader contribution centered at 532.7, which is characteristic of hydrogenbonded carboxylic groups (with contributions from both hydroxy and carbonyl oxygens ${ }^{11-13}$ ). This observation together with the XPS data for the MOCC-S phase shown in section IV A is in agreement with the RAIRS results; up to a coverage of 0.53 $\mathrm{ML}$, the acid groups deprotonate and form carboxylate- copper complexes; at higher coverages, the TMA molecule adsorbs with at least one group remaining intact in the acidic form.

The situation changes upon heating to $500 \mathrm{~K}$, as the layer contains exclusively carboxylate groups, as seen by the disappearance of the $289.4 \mathrm{eV}$ component in the $\mathrm{C} 1 \mathrm{~s}$ spectrum and of the $532.7 \mathrm{eV}$ component in the $\mathrm{O} 1 \mathrm{~s}$ spectrum (Figure $17 \mathrm{~b}$ and $\mathrm{d}$, respectively). A detailed inspection of the $\mathrm{Cu} 2 \mathrm{p}_{3 / 2}$ spectra in Figure 17e and $\mathrm{f}$ further supports the picture obtained from the STM and DFT analysis. For a coverage of $1 \mathrm{ML}$, the chemically shifted component at $933.6 \mathrm{eV}$ corresponding to a $\mathrm{Cu}-\mathrm{O}$ bond has a similar binding energy as that in the lowcoverage phase (cf. Figure 4d). However, upon heating, the shift is increased from 1.4 to $2.0 \mathrm{eV}$. This larger shift could be explained by the higher oxygen coordination of the $\mathrm{Cu}$ adatoms 


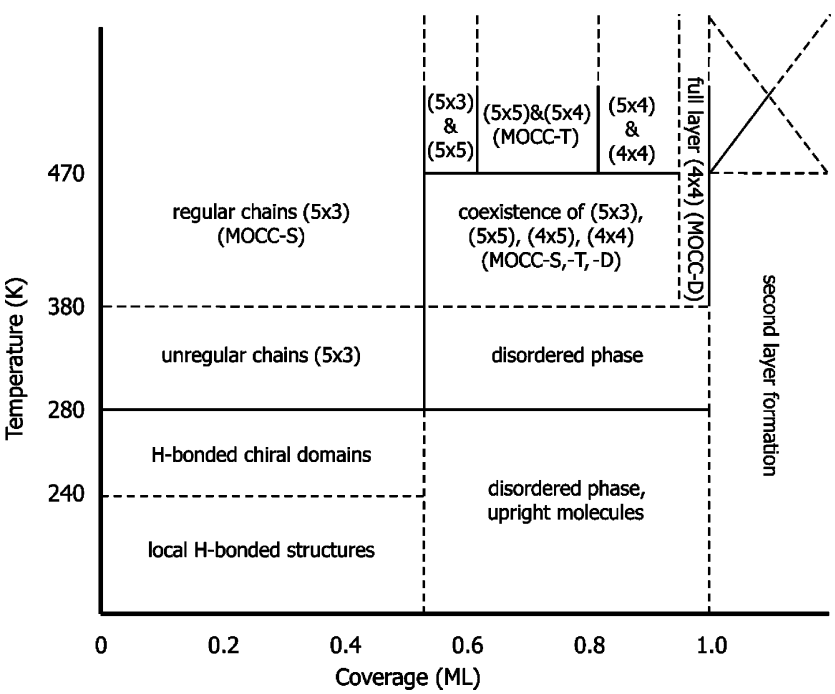

Figure 18. Phase diagram for the adsorption of TMA on $\mathrm{Cu}(110)$.

in the MOCC-D phase as determined by the DFT simulations (two carboxylate groups bound to one $\mathrm{Cu}$ adatom, cf. Figure 16).

\section{Conclusions and Perspectives}

The data presented in this study can be used to construct an adsorption phase diagram ${ }^{4}$ for the TMA/Cu(110) system as shown in Figure 18.

The horizontal line at $280 \mathrm{~K}$ represents a relatively narrow temperature window of about 20 degrees separating two markedly different structural organizations. At temperatures below this threshold, the adsorbed molecules are only partially deprotonated, and therefore, intermolecular interactions are mainly dominated by $\mathrm{H}$ bonding. Above this temperature, the molecules are completely deprotonated, and their interaction is mediated by metal-organic coordination bonding. This transition is driven by the emission of $\mathrm{Cu}$ adatoms from lowcoordinated surface sites. Its narrow temperature range is due to the activated nature of these processes, and the temperature at which it is occurring is in good agreement with that predicted from the calculated energy barriers. ${ }^{79}$ The availability of extremely reactive and low-coordinated $\mathrm{Cu}$ adatoms has a dual effect, it substantially accelerates the deprotonation process, ${ }^{12}$ and it furnishes the metallic centers needed for the formation of the metal-organic structures.

A comparison of the adsorption of TMA on $\mathrm{Cu}(110)$ and (001) surfaces shows distinct similarities. On $\mathrm{Cu}(001)$ at low substrate temperatures, a strong $\mathrm{H}$-bonding tendency of the intact acid groups leads to the formation of honeycomb structures. Partial deprotonation starts above $300 \mathrm{~K}$, but the complete transition to the trimesate molecular form and to the formation of metal-organic structures occurs only after heating to $400 \mathrm{~K}$ or higher. ${ }^{24}$ This difference in the activation temperatures for both the deprotonation and the formation of metal-organic complexes can be explained by the higher energy barriers of the corresponding processes (loss of hydrogen atoms and emission of $\mathrm{Cu}$ adatoms, respectively) on the $\mathrm{Cu}(001)$ surface. ${ }^{7,12}$

Figure 18 shows the rich variety of the adsorption phase diagram for the TMA/Cu(110) system. This complexity stems from the multifunctionality of the elementary molecular building blocks and from a fine balance between surface-molecule and molecule-molecule interactions in the total energy minimization. The very detailed characterization we have presented in this paper was only possible thanks to a combined application of different and complementary experimental and theoretical investigation techniques. We believe that, although the details of this system may depend on the particular choice of molecule and substrate, other aspects such as the temperature- and substrate-dependent deprotonation, the role of adatoms, the templating effect of the substrate, and the interplay between packing and formation energy are still quite general. As a consequence, the precise analysis presented here might be very useful for the comprehension of other supramolecular systems at surfaces, particularly for those involving carboxylic functional groups. ${ }^{6-8,10,24}$

Acknowledgment. The authors wish to acknowledge Nian Lin, Alexander Schneider, and Riccardo Ferrando for fruitful discussions. Y.W. acknowledges the Alexander von Humboldt foundation for financial support.

\section{References and Notes}

(1) Barth, J. V.; Costantini, G.; Kern, K. Nature 2005, 437, 671

(2) Schreiber, F. Prog. Surf. Sci. 2000, 65, 151.

(3) Rosei, F.; Schunack, M.; Naitoh, Y.; Jiang, P.; Gourdon, A.; Laegsgaard, E.; Stensgaard, I.; Joachim, C.; Besenbacher, F. Progr. Surf Sci. 2003, 71, 95 .

(4) Barlow, S. M.; Raval, R. Surf. Sci. Rep. 2003, 50, 201.

(5) Ruben, M.; Rojo, J.; Romero-Salguero, F. J.; Uppadine, L. H.; Lehn, J.-M. Angew. Chem., Int. Ed. 2004, 43, 3644. 292,33

(6) Frederick, B. G.; Ashton, M. R.; Richardson, N. V. Surf. Sci. 1993,

(7) Perry, C. C.; Haq, S.; Frederick, B. G.; Richardson, N. V. Surf. Sci. 1998, 409, 512 .

(8) Chen, Q.; Perry, C. C.; Frederick, B. G.; Murray, P. W.; Haq, S.; Richardson, N. V. Surf. Sci. 2000, 446, 63.

(9) Martin, D. S.; Cole, R. J.; Haq, S. Surf. Sci. 2003, 539, 171.

(10) Martin, D. S.; Cole, R. J.; Haq, S. Phys. Rev. B 2002, 66, 155427.

(11) Stepanow, S.; Strunskus, T.; Lingenfelder, M.; Dmitriev, A.; Spillmann, H.; Lin, N.; Barth, J. V.; Wöll, C.; Kern, K. J. Phys. Chem. B 2004, 108, 19392

(12) Lin, N.; Payer, D.; Dmitriev, A.; Strunskus, T.; Wöll, C.; Barth, J.; Kern, K. Angew. Chem., Int. Ed. 2005, 44, 1488.

(13) Dmitriev, A.; Spillmann, H.; Stepanow, S.; Strunskus, T.; Woell, C.; Seitsonen, A.; Lingenfelder, M.; Lin, N.; Barth, J.; Kern, K. ChemPhysChem 2006, 7, 2197.

(14) Payer, D.; Comisso, A.; Dmitriev, A.; Strunskus, T.; Lin, N.; Woell, C.; DeVita, A.; Barth, J. V.; Kern, K. Chem.-Eur. J. 2007, 13, 3900.

(15) Férey, G. Chem. Mater. 2001, 13, 3084

(16) Cheetham, A. K.; Férey, G.; Loiseau, T. Angew. Chem., Int. Ed. 1999, 38, 3268

(17) Desiraju, G. R. Angew. Chem., Int. Ed. Engl. 1995, 34, 2311

(18) Yaghi, O. M.; O'Keeffe, M.; Ockwig, N. W.; Chae, H. K.; Eddaoudi, M.; Kim, J. Nature 2003, 423, 705.

(19) Kitagawa, S.; Kitaura, R.; Noro, S. Angew. Chem., Int. Ed. 2004, $43,2334$.

(20) Swiegers, G. F.; Malefetse, T. J. Chem. Rev. 2000, 100, 3483.

(21) Holliday, B. J.; Mirkin, C. A. Angew. Chem., Int. Ed. 2001, 40, 2022 .

(22) Chae, H. K.; Siberio-Pérez, D. Y.; Kim, J.; Go, Y.; Eddaoudi, M.; Matzger, A. J.; O’Keefe, M.; Yaghi, O. M. Nature 2004, 427, 523.

(23) Prestipino, C.; Regli, L.; Vitillo, J. G.; Bonino, F.; Damin, A.; Lamberti, C.; Zecchina, A.; Solari, P. L.; Kongshaug, K. O.; Bordiga, S. Chem. Mater. 2006, 18, 1337.

(24) Dmitriev, A.; Lin, N.; Weckesser, J; Barth, J. V.; Kern, K. J. Phys. Chem. B 2002, 106, 6907.

(25) Lin, N.; Dmitriev, A.; Weckesser, J.; Barth, J. V.; Kern, K. Angew. Chem., Int. Ed. 2002, 41, 4779.

(26) Messina, P.; Dmitriev, A.; Lin, N.; Spillmann, H.; Abel, M.; Barth, J. V.; Kern, K. J. Am. Chem. Soc. 2002, 124, 14000.

(27) Dmitriev, A.; Spillmann, H.; Lin, N.; Barth, J. V.; Kern, K. Angew. Chem., Int. Ed. 2003, 42, 2670.

(28) Spillmann, H.; Dmitriev, A.; Lin, N.; Messina, P.; Barth, J. V.; Kern, K. J. Am. Chem. Soc. 2003, 125, 10725.

(29) Lingenfelder, M. A.; Spillmann, H.; Dmitriev, A.; Stepanow, S.; Lin, N.; Barth, J. V.; Kern, K. Chem.-Eur. J 2004, 10, 1913.

(30) Stepanow, S.; Lingenfelder, M.; Dmitriev, A.; Spillmann, H.; Delvigne, E.; Lin, N.; Deng, X.; Cai, C.; Barth, J. V.; Kern, K. Nat. Mater. 2004, 3, 229.

(31) Dmitriev, A.; Spillmann, H.; Lingenfelder, M.; Lin, N.; Barth, J. V.; Kern, K. Langmuir 2004, 20, 4799.

(32) Ruben, M. Angew. Chem., Int. Ed. 2005, 44, 1594. 
(33) Stepanow, S.; Lin, N.; Vidal, F.; Landa, A.; Ruben, M.; Barth, J. V.; Kern, K. Nano Lett. 2005, 5, 901.

(34) Classen, T.; Fratesi, G.; Costantini, G.; Fabris, S.; Stadler, F. L.; Kim, C.; de Gironcoli, S.; Baroni, S.; Kern, K. Angew. Chem., Int. Ed. 2005, 44, 6142 .

(35) Gambardella, P.; Stepanow, S.; Dmitriev, A.; Gupta, S. S.; Honolka, J.; Clair, S.; Pons, S.; Lingenfelder, M.; Bencok, P.; Stanescu, S. et al. 2007, submitted.

(36) Semenov, A.; Spatz, J. P.; Möller, M.; Lehn, J.-M.; Sell, B.; Schubert, D.; Weidl, C. H.; Schubert, U. S. Angew. Chem., Int. Ed. 1999, $38,2547$.

(37) Ziener, U.; Lehn, J.-M.; Mourran, A.; Möller, M. Chem.-Eur. J. 2002, 8, 951 .

(38) Lin, N.; Stepanow, S.; Vidal, F.; Barth, J. V.; Kern, K. Chem. Commun. 2005, 1681.

(39) Vidal, F.; Delvigne, E.; Stepanow, S.; Lin, N.; Barth, J. V.; Kern, K. J. Am. Chem. Soc. 2005, 127, 10101.

(40) Lehn, J.-M. Proc. Natl. Acad. Sci. U.S.A. 2002, 99, 4763.

(41) Weissbuch, I.; Baxter, P. N. W.; Kuzmenko, I.; Cohen, H.; Cohen, S.; Kjaer, K.; Howes, P. B.; Als-Nieslsen, J.; Lehn, J.-M.; Leiserowitz, L.; Lahay, M. Chem.-Eur. J. 2000, 6, 725.

(42) Kurth, D. G.; Severin, N.; Rabe, J. P. Angew. Chem., Int. Ed. 2002, 41,3681 .

(43) de Feyter, S.; Abdel-Mottaleb, M. M. S.; Schuurmans, N.; Verkuijl, B. J. V.; van Esch, J. H.; Feringa, B. L.; de Schryver, F. C. Chem.-Eur. J. 2004, 10, 1124.

(44) Panella, B.; Hirscher, M.; Pütter, H.; Müller, U. Adv. Funct. Mater. 2006, 16,520 .

(45) Lehn, J.-M. Supramolecular Chemistry; VCH: Weinheim, Germany, 1995; Chapter 9, p 200.

(46) Duchamp, D.; Marsh, R. E. Acta. Crystallogr., Sect. B 1969, 25, 5 .

(47) Cheng, D.; Khan, M. A.; Houser, R. P. Inorg. Chem. 2001, 40, 6858.

(48) Chui, S. S.-Y.; Lo, S. M.-F.; Charmant, J. P. H.; Orpen, A. G.; Williams, I. D. Science 1999, 283, 1148.

(49) Griessl, S.; Lackinger, M.; Edelwirth, M.; Hietschold, M.; Heckl,

W. M. Single Mol. 2002, 3, 25.

(50) Lackinger, M.; Griessl, S.; Kampschulte, L.; Jamitzky, F.; Heckl, W. M. Small 2005, 5, 532.

(51) Su, G.-J.; Zhang, H.-M.; Wan, L.-J.; Bai, C.-L.; Wandlowski, T. J. Phys. Chem. B 2004, 108, 1931. 6915.

(52) Li, Z.; Han, B.; Wan, L. J.; Wandlowski, T. Langmuir 2005, 21,
(53) Ishikawa, Y.; Ohira, A.; Sakata, M.; Hirayama, C.; Kunitake, M. Chem. Commun. 2002, 22, 2652.

(54) Sheerin, G.; Cafolla, A. A. Surf. Sci. 2005, 577, 211.

(55) Nath, K. G.; Ivasenko, O.; Miwa, J. A.; Dang, H.; Wuest, J. D.;

Nanci, A.; Perepichka, D. F.; Rosei, F. J. Am. Chem. Soc. 2006, 128, 4212 (56) Wang, Q.-L.; Liang, M.; Liao, D.-Z.; Yan, S.-P.; Jiang, Z.-H.;

Cheng, P. Z. Anorg. Allg. Chem. 2004, 630, 613.

(57) Konar, S.; Mukherjee, P. S.; Zangrando, E.; Drew, M. G. B.; Diaz,

C.; Ribas, J.; Chaudhuri, N. R. Inorg. Chim. Acta 2005, 358, 29.

(58) Mahowald, P. H.; Friedman, D. J.; Carey, G. P.; Bertness, K. A.; Yeah, J. J. J. Vac. Sci. Technol., A 1987, 5, 2982.

(59) Perdew, J.; Burke, K.; Ernzerhof, M. Phys. Rev. Lett. 1996, 77, 3865 .

(60) Vanderbilt, D. Phys. Rev. B 1990, 41, 7892

(61) Baroni, S.; Corso, A. D.; de Gironcoli, S.; Giannozzi, P. Plane-

Wave Self-Consistent Field Home Page. http://www.pwscf.org.

(62) Tersoff, J.; Hamann, D. R. Phys. Rev. Lett. 1983, 50, 1998.

(63) Han, B.; Li, Z.; Pronkin, S.; Wandlowski, T. Can. J. Chem. 2004 82,1481 .

(64) Han, B.; Li, Z.; Wandlowski, T. Anal. Bioanal. Chem. 2007, 388, 121.

(65) Gonzáles-Sánchez, F. Spectrochim. Acta 1958, 12, 17.

(66) Arenas, J. F.; Montanez, M. A.; Otero, J. C.; Marcos, J. I. Vib. Spectrosc. 1993, 4, 159.

(67) Usuki, N. Vacuum 1990, 41, 1683.

(68) Chen, C. J. Phys. Rev. Lett. 1992, 69, 1656.

(69) Chen, C. J. J. Vac. Sci. Technol., B 1994, 12, 2193.

(70) Barth, J. V.; Brune, H.; Ertl, G. Phys. Rev. B 1990, 42, 9307.

(71) Coulman, D. J.; Wintterlin, J.; Behm, R. J.; Ertl, G. Phys. Rev.

Lett. 1990, 64, 1761 .

(72) Kern, K.; Niehus, H.; Schatz, A.; Zeppenfeld, P.; Goerge, J.; Comsa, G. Phys. Rev. Lett. 1991, 67, 855 .

(73) Jensen, F.; Besenbacher, F.; Laegsgaard, E.; Stensgaard, I. Phys. Rev. B 1990, 42, 9206.

(74) Doyle, A.; Felcman, J.; do Prado Gambardella, M. T.; Verani, C. N.; Tristão, M. L. B. Polyhedron 2000, 19, 2621.

(75) Bae, C.; Freeman, D. L.; Doll, J. D.; Kresse, G.; Hafner, J. J. Chem

Phys. 2000, 113, 6926.

(76) Deacon, G. B.; Phillips, M. J. Coord. Chem. Rev. 1980, 33, 227.

(77) Desiraju, G. R. Acc. Chem. Res. 1996, 29, 441

(78) Steiner, T. Angew. Chem., Int. Ed. 2002, 41, 48.

(79) Mottet, C.; Ferrando, R.; Hontinfinde, F.; Levi, A. C. Surf. Sci 1998, 417, 220 . 